\title{
Direct numerical simulations of transition in a compressor cascade: the influence of free-stream turbulence
}

\author{
TAMER A. ZAKI ${ }^{1} \dagger$, JAN G. WISSINK ${ }^{2}$, WOLFGANG RODI \\ AND PAUL A. DURBIN ${ }^{4}$ \\ ${ }^{1}$ Department of Mechanical Engineering, Imperial College London, Exhibition Road, \\ London SW7 2AZ, UK \\ ${ }^{2}$ School of Engineering and Design, Brunel University, Uxbridge, UB8 3PH, UK \\ ${ }^{3}$ Institute for Hydromechanics, Karlsruhe Institute of Technology, \\ Kaiserstr. 12, D-76128 Karlsruhe, Germany \\ ${ }^{4}$ Aerospace Engineering, Iowa State University, Ames, IA 50011, USA
}

(Received 9 November 2009; revised 14 July 2010; accepted 14 July 2010; first published online 27 October 2010)

The flow through a compressor passage without and with incoming free-stream grid turbulence is simulated. At moderate Reynolds number, laminar-to-turbulence transition can take place on both sides of the aerofoil, but proceeds in distinctly different manners. The direct numerical simulations (DNS) of this flow reveal the mechanics of breakdown to turbulence on both surfaces of the blade. The pressure surface boundary layer undergoes laminar separation in the absence of free-stream disturbances. When exposed to free-stream forcing, the boundary layer remains attached due to transition to turbulence upstream of the laminar separation point. Three types of breakdowns are observed; they combine characteristics of natural and bypass transition. In particular, instability waves, which trace back to discrete modes of the base flow, can be observed, but their development is not independent of the Klebanoff distortions that are caused by free-stream turbulent forcing. At a higher turbulence intensity, the transition mechanism shifts to a purely bypass scenario. Unlike the pressure side, the suction surface boundary layer separates independent of the freestream condition, be it laminar or a moderate free-stream turbulence of intensity $T_{u} \sim 3 \%$. Upstream of the separation, the amplification of the Klebanoff distortions is suppressed in the favourable pressure gradient (FPG) region. This suppression is in agreement with simulations of constant pressure gradient boundary layers. FPG is normally stabilizing with respect to bypass transition to turbulence, but is, thereby, unfavourable with respect to separation. Downstream of the FPG section, a strong adverse pressure gradient (APG) on the suction surface of the blade causes the laminar boundary layer to separate. The separation surface is modulated in the instantaneous fields of the Klebanoff distortion inside the shear layer, which consists of forward and backward jet-like perturbations. Separation is followed by breakdown to turbulence and reattachment. As the free-stream turbulence intensity is increased, $T_{u} \sim 6.5 \%$, transitional turbulent patches are initiated, and interact with the downstream separated flow, causing local attachment. The calming effect, or delayed re-establishment of the boundary layer separation, is observed in the wake of the turbulent events.

Key words: boundary layers, bypass transition, compressor cascade, pressure gradient 


\section{Introduction}

Transition induced by free-stream turbulence is of avid interest due to its role in turbo-machinery flows (Hodson \& Howell 2005). It is also of fundamental interest as an example of what is called bypass transition. Therefore, both canonical configurations, such as flat-plate boundary layers, and more realistic turbine passages have been previously investigated; the latter often uncovering new fundamental transition scenarios. For example, the simulations of $\mathrm{Wu} \&$ Durbin (2001) demonstrated the formation of elongated, streamwise vortices on the pressure surface of a turbine blade. Despite their formation on the concave surface of the blade, they are not of the Görtler type, but rather induced by the distorted migrating wakes. As we show herein, the compressor geometry proves to be even richer in fundamental transition phenomena under free-stream turbulence.

Recent theoretical studies and computer simulations have provided a basic understanding of some of the phenomena of transition under vortical perturbations. However, the influence of free-stream turbulence upon transition in streamwise pressure gradients remains uncertain. A net pressure rise could promote transition, but the outcome is dependent on the pressure gradient history (Gostelow, Blunden \& Walker 1994); the pressure rise only ensures transition by the end of the aerofoil. From what is known of bypass transition, even that is likely to be altered by free-stream turbulence. The interaction of pressure distribution and the forcing from the free stream raises many possibilities: orderly transition via inflectional instability modes, bypass via Klebanoff distortions, lambda vortices forced by an interaction between streaks and instability waves, instability of separated layers. These are possibilities that appear in the existing literature and could exist in the compressor geometry. However, they are not exhaustive and their relevance is uncertain. In the computations presented here, some of the above processes are observed, depending on the intensity of the freestream turbulence, with the pressure and suction sides showing a markedly different behaviour.

An exhaustive account of all the potentially relevant transition processes must consider the nature of the free-stream vortical disturbance, leading-edge effects, pressure gradient and surface curvature. The discussion of each relevant component is deferred to the appropriate sections of the paper. In this introduction, a brief overview is provided of canonical transition mechanisms that are relevant to this work.

\subsection{Orderly transition}

The orderly route to boundary-layer turbulence begins with the amplification of linear instability waves. In zero-pressure-gradient boundary layers, these are viscous Tollmien-Schlichting (T-S) waves which are two-dimensional at the critical Reynolds number, $R e=\sqrt{U x / v} \approx 270$. As the Reynolds number is increased, the $\mathrm{T}-\mathrm{S}$ waves amplify, but transition is not certain. Once the Reynolds number crosses the upper branch of the stability curve, the linear instability waves return to a stable state. Transition only takes place if the instability waves reach an amplitude of the order of $1 \%$ of the free-stream speed, before crossing the upper branch. This amplitude marks the onset of secondary instability, which leads rapidly to breakdown into turbulence.

The secondary instability is three-dimensional. It is attributed to a parametric excitation of the base flow, which now includes the finite-amplitude $\mathrm{T}-\mathrm{S}$ distortion (Herbert 1988). Spanwise-periodic $\Lambda$-patterns develop on top of the T-S waves (Klebanoff, Tidstrom \& Sargent 1962). The arrangement from row to row can be aligned (K-type) due to fundamental resonance, or staggered (C-type and H-type) due to subharmonic resonance. Beyond the secondary instability stage, nonlinear 
breakdown has also been investigated empirically, using detailed experiments and numerical simulations (see for example Kleiser \& Zang 1991).

\subsection{Bypass transition}

Investigations of boundary-layer transition beneath vortical disturbances have yielded a wealth of interesting mechanisms. Some are influenced by the geometry of the leading edge (Kendall 1991), by the mean pressure gradient and its history (AbuGhannam \& Shaw 1980; Gostelow et al. 1994), or by the turbulence intensity and length scale (Jacobs \& Durbin 2000; Brandt, Schlatter \& Henningson 2004; Ovchinnikov, Choudhari \& Piomelli 2008). To study bypass transition, detailed characterization of the disturbance field is required, for example the level of anisotropy and decay rate of the free-stream vortical disturbances which force the boundary layer (Westin et al. 1994; Matsubara \& Alfredsson 2001).

In the absence of leading-edge and significant pressure gradient effects, bypass transition proceeds by amplification of the well-known Klebanoff distortions (Klebanoff et al. 1962; Morkovin 1969). These streamwise-elongated disturbances are forced by the low-frequency component of free-stream turbulence. It is known from linear theory that the boundary layer acts as a low-pass filter, admitting lowfrequency perturbations from the free stream (Jacobs \& Durbin 2000; Zaki \& Saha 2009). Shear causes these to amplify due to the 'lift up' mechanism (Phillips 1969), thus forming Klebanoff distortions. The flow in these 'streaks' is dominated by the $u$-component, in a region of long streamwise extent. The amplification of Klebanoff distortions and the 'universality' of their wall-normal profile are well documented (Westin et al. 1994; Matsubara \& Alfredsson 2001). Their spanwise scale is explained by a competition between the shear amplification mechanism and viscous decay, and is well predicted by non-modal growth analyses (Butler \& Farrell 1992; Andersson, Berggren \& Henningson 1999; Luchini 2000).

In pure bypass transition, the low-speed streaks lift from the surface and initiate transition near the top of the boundary layer. Higher frequencies in the free stream penetrate the upper part of the boundary layer, the penetration depth decreasing with increasing frequency. Higher frequencies trigger secondary instabilities of Klebanoff distortions and lead to transition. At least, that is the perspective of a localized interaction, which was observed in the direct numerical simulations (DNS) by Jacobs \& Durbin (2000) at free-stream turbulence intensity $T_{u} \sim 3.5 \%$, and verified experimentally by Hernon, Walsh \& McEligot (2007).

Distortion at the leading edge provides another potential route for coupling vortical disturbances to the boundary layer. For example, Nagarajan, Lele \& Ferziger (2007) contrasted transition in the presence of slender and blunt leading edges, when the free-stream turbulence intensity is $T_{u} \sim 3.5-4.5 \%$. The blunt leading edge led to the formation of instability wavepackets, which amplified downstream and caused breakdown to turbulence. While they could trace the origin of the packet to the leading edge, the instability mechanism could not be unambiguously identified. More recently, Goldstein \& Sescu (2008) showed that the bluntness of the leading edge can lead to the amplification of small-amplitude, unsteady disturbances. Downstream, the base flow becomes inflectional and inviscidly unstable.

A recent simulation of the boundary-layer transition on a flat plate addressed the interaction of Klebanoff modes on T-S waves (Liu, Zaki \& Durbin 2008a). A mixture of the pure orderly and bypass routes was observed. The fluctuating streamwise jets, which compose the Klebanoff distortion, warp the instability wavefronts, inducing transition through $\Lambda$-vortices. The interaction is, however, complex. A 
steady distortion has a stabilizing influence on T-S waves - a view supported by analyses (Cossu \& Brandt 2002) and experimental observations (Fransson et al. 2005). However, an unsteady Klebanoff distortion can also enhance the secondary instability of T-S waves, and thus promote transition (Liu, Zaki \& Durbin 2008b).

The relevance of these canonical simulations to compressor blades is uncertain: in zero pressure gradient, the $\mathrm{T}-\mathrm{S}$ waves have a very weak exponential growth rate and are not observed under free-stream turbulence of about $1 \%$. Two locations where the interaction of Klebanoff distortions and $\mathrm{T}-\mathrm{S}$ waves can become relevant are near a blunt leading edge (Kendall 1991) and in an adverse pressure gradient. The latter was reported by Hughes \& Walker (2001), who used wavelet transforms to extract instability waves from surface stress measurements on the suction surface of a compressor blade $\left(R e=1.1-1.3 \times 10^{5}, T_{u} \sim 2-3 \%\right)$. The high-pass filtered data yielded evidence of growing instability waves, which they asserted could precede the appearance of turbulent spots.

\subsection{The influence of strong adverse pressure gradient}

In adverse pressure gradient, the boundary-layer profile becomes inflectional. It is therefore prone to inviscid instability, which has significantly larger growth rates than viscous, zero-pressure-gradient $\mathrm{T}-\mathrm{S}$ waves. In addition, discrete mode triad interactions have higher amplification rates in decelerated flow (Goldstein \& Lee 1992; Corke \& Gruber 1996). The threshold for orderly transition is thus reduced.

A summary of experimental investigations of bypass transition in pressure gradients is provided by Abu-Ghannam \& Shaw (1980). They show that the location of transition is more sensitive to adverse pressure gradient than to favourable pressure gradient. However, that sensitivity is reduced when the free-stream turbulence intensity is higher than $3 \%$. In a more recent study, Gostelow et al. (1994) demonstrated that transition is affected by the history of the pressure gradient.

In highly adverse pressure gradients, the boundary layer is at risk of separation which, in the presence of free-stream vortical forcing, is followed by a rapid transition to turbulence. Numerical simulations that fully resolve separation-induced transition were conducted by Alam \& Sandham (2000) and Spalart \& Strelets (2000). Both papers report on DNS of flow over a flat plate where suction is applied at the upper boundary in order to induce separation. The linear stability analysis by Alam \& Sandham (2000) indicated that absolute instability of the separation bubble occurs when the reverse flow is greater than $15 \%$. Their simulations also predicted a $\Lambda$ vortex-induced breakdown to turbulence of the separated shear layer. On the other hand, the simulation of Spalart \& Strelets (2000) did not yield clear evidence of primary or secondary instability, and the flow very rapidly became three-dimensional. In both simulations, the turbulence was observed to be sustained even though the flow was not absolutely unstable.

The above studies are all concerned with the influence of pressure gradient on flat-plate boundary layers. More recently, DNS of transition in realistic aerofoil geometries was carried out. In a compressor passage, both separation-induced and bypass transitions are likely to be promoted, due to the overall flow deceleration. These mechanisms were observed even in DNS of flow through turbine passages, where the overall pressure gradient is favourable. Wu \& Durbin (2001) simulated flow in a T106 turbine cascade with periodically incoming wakes, at $R e=1.48 \times 10^{5}$. They observed that the incoming wakes are distorted as they convect through the passage. On the suction surface, turbulent spots are observed near the rear of the blade, in the adverse-pressure-gradient section of the aerofoil. At larger angle of 
attack, Wissink (2003) and Wissink, Rodi \& Hodson (2006) studied the influence of migrating wakes on the laminar separation bubble, albeit at a lower Reynolds number $(R e=51,831)$. A Kelvin-Helmholtz $(\mathrm{K}-\mathrm{H})$ instability was observed to be triggered by the periodic passing of the wakes. Small-scale fluctuations inside the wake were found to be responsible for triggering further transition to turbulence inside the $\mathrm{K}-\mathrm{H}$ rolls.

Jones, Sandberg \& Sandham (2008) reported on simulations of flow over a NACA-0012 aerofoil at $5^{\circ}$ incidence, and $R e=5 \times 10^{4}$. A laminar separation bubble originating at approximately $15 \%$ axial chord was the seat of breakdown to turbulence. The flow downstream of the transition location was intermittently separated due to the passing of the shed vortices. The two-dimensional vortex shedding was found to be absolutely unstable to three-dimensional perturbations. A mechanism was proposed - depending on strong local reverse flow and large instability growth rates in braid regions - by which the instability could sustain itself.

A number of studies of transitional Reynolds number flows in compressor passages have appeared in the experimental literature. Hughes \& Walker (2001) investigated the presence of natural transition within the boundary layer on the suction surface of a compressor blade at $R e=1.1-1.3 \times 10^{5}$. The blade was a stator, downstream of a 1.5 stage low-speed compressor cascade. While their Reynolds number was below the normal operating range in conventional aircraft engines $\left(R e \geqslant 6 \times 10^{5}\right)$, it was above the critical values for laminar separation to cause significant losses. Other investigations were concerned with near-critical conditions, and the influence of freestream turbulence on the flow dynamics (Schreiber et al. 2004; Sonoda et al. 2004). Such investigations are important for characterizing the off-design performance of conventional engines, and also the design of smaller engines. For example, Sonoda et al. (2004) focused on the compressor blade design for the outlet guide vanes (OGV) of small aircraft turbofan engines. Their design operated at transitional Reynolds number of approximately $1.3 \times 10^{5}$ and with a turning angle of $43^{\circ}$ (similar to our DNS) at the cruise point. Under these conditions, they showed that significant losses can emerge below a critical Reynolds number $2 \times 10^{5}$, due to flow separation. Therefore, transition plays a significant role in the blade design in this regime.

The current simulations combine curvature, pressure gradient and free-stream turbulence effects. Various intensities of free-stream forcing and their influence on transition in a NACA 65 compressor geometry are considered. The pressure side experiences an adverse pressure gradient along most of its forward portion. Nevertheless, at the Reynolds number of the present simulation, transition would be quite slow in the absence of free-stream perturbations. It will be shown that free-stream turbulence alters the boundary layer radically.

The next section, $\S 2$, describes the computational set-up and the flow without incoming turbulence. This provides the baseline against which to judge the effect of free-stream turbulence. The ensuing sections provide our detailed observations and computational data.

\section{The simulation set-up}

Figure 1 is a schematic of the computational domain. The blade geometry is designated NACA 65. The linear low-pressure (LP) compressor cascade in figure 1 is largely in accordance with experiments performed at the University of the Armed Forces in Munich (Hilgenfeld \& Pfitzner 2004). The linear cascade in the experiments 


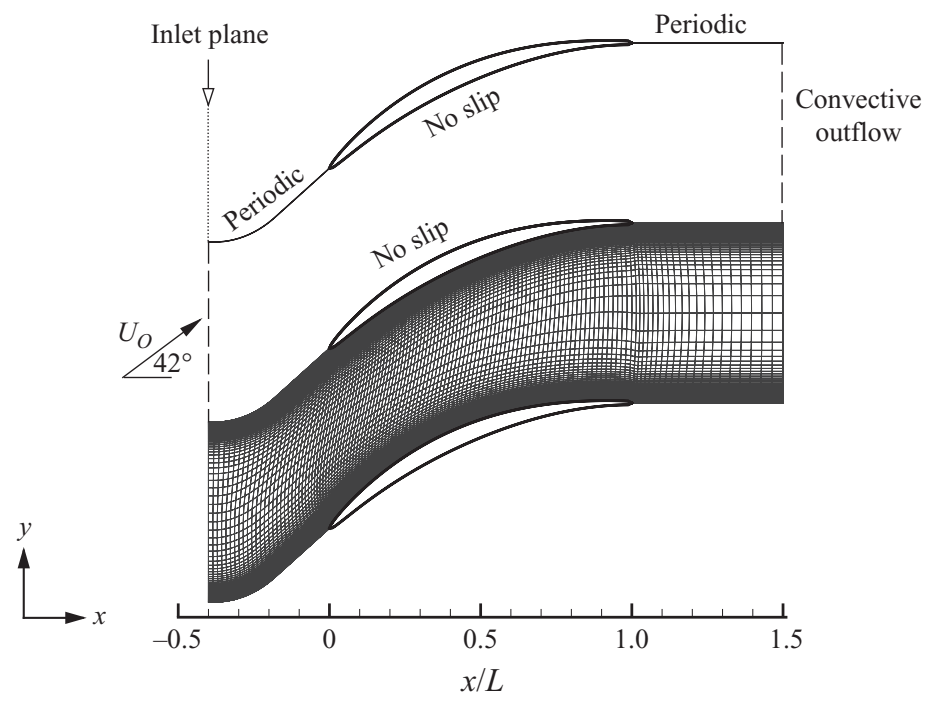

FIGURE 1. Upper part: cross-section through the computational domain at mid-span. Lower part: computational grid showing every eighth line in $x$ and $y$.

was denoted V103. In the simulations, only one of the passages is calculated, with periodic boundary conditions in the $y$-direction, upstream and downstream of the blade surface - to account for the cascade. The periodic regions are $x / L<0$ and $x / L>1$, where the axial chord $L$ is selected as the characteristic length scale. The mean inflow speed $U_{0}$ is chosen as the characteristic velocity. Based on these reference scales, the simulation Reynolds number is $R e=138,500$.

The periodic condition in the $y$-direction has been widely adopted in the previous DNS of flow in turbine passages, for example, by Wu \& Durbin (2001) and Wissink \& Rodi (2006). This periodicity, however, implies synchronous shedding of trailing-edge vortices from all the blades in a row. Abdessemed, Sherwin \& Theofilis (2009) studied this effect in the context of flow stability within turbine passages, at a relatively low Reynolds number $\operatorname{Re} \sim 5 \times 10^{3}$. Using two passages instead of one, they showed that the shedding can become asynchronous from two consecutive blades in the cascade. The phase shift was not appreciable, but affected the flow stability. This was primarily due to the strong turning of the flow in the turbine passage: the unit normal to the suction surface bubble of one blade intersected the trailing-edge vortices from the blade above in their T106 configuration. In the current flow set-up, the exit velocity is nearly horizontal, and the trailing-edge wake does not interfere with the upstream flow.

The top and bottom computational boundaries are separated by one blade pitch, $P=0.59 \mathrm{~L}$. The streamwise extent of the domain is $1.9 \mathrm{~L}$. The inflow plane is $x / L=-0.4$, at which a mean velocity $\left(U_{o} \cos (\alpha), U_{o} \sin (\alpha), 0\right)$ is prescribed; the inflow angle is $\alpha=42^{\circ}$. Convective boundary conditions are applied at the outflow, $x / L=1.5$ and periodic boundary conditions are enforced in the spanwise direction. The spanwise size of the domain is $0.20 \mathrm{~L}$, which is approximately 10 times the maximum boundary-layer thickness. In the experiments (Hilgenfeld \& Pfitzner 2004), both periodically incoming wakes and free-stream turbulence were introduced in the inflow region of the cascade. The focus here is on the influence of the incoming turbulence intensity on the dynamics of the flow. 


$\begin{array}{ccc}\text { Simulation } & \text { Grid } & T_{u} \text { at } x / L=-0.4 \\ \text { T0 } & 1025 \times 641 \times 65 & 0.00 \\ \text { T1 } & 1025 \times 641 \times 129 & 3.25 \\ \text { T2 } & 1025 \times 641 \times 129 & 6.50 \\ \text { T3 } & 1025 \times 641 \times 129 & 8.00 \\ \text { T4 } & 1025 \times 641 \times 129 & 10.00\end{array}$

TABLE 1. Overview of the direct numerical simulations performed. $T_{u}$ is the turbulence intensity.

The incompressible Navier-Stokes equations are discretized on a staggered grid, with a local volume flux formulation (Rosenfeld, Kwak \& Vinokur 1991; Wu \& Durbin 2001). Time advancement of the convective terms is by second-order AdamsBashforth. The pressure and diffusion terms are treated using implicit Euler and Crank-Nicolson, respectively.

In post-processing, flow quantities are decomposed into a time-averaged mean and a perturbation component, e.g. $\boldsymbol{u}=\overline{\boldsymbol{u}}+\boldsymbol{u}^{\prime}$, and the streamwise and wall-normal coordinates are denoted by the subscripts $t$ and $n$, respectively.

Initially, a laminar simulation, without inflow perturbations, was carried out on a coarse mesh comprising $625 \times 289 \times 65$ points in the $x, y$ and spanwise directions, respectively. The same $(x, y)$ grid was used for a preliminary simulation with inflow turbulence. Based on the results from the coarse mesh computations, the grid was refined in order to fully resolve the boundary layer on both the pressure and suction sides. This exercise was particularly important on the suction surface due to the presence of a thin separation bubble. The final, fine mesh is composed of $1025 \times 641 \times 129$ points $(84.76$ million). It provides a higher resolution outside the boundary layers in order to avoid any excessive decay of the free-stream turbulence. Figure 1 illustrates the final mesh, with only every eighth grid line plotted. The grid resolution at the blade surface in the tangent, normal and spanwise directions is $5<\Delta_{t}^{+}<10,0.5<\Delta_{n}^{+}<1.0$ and $5<\Delta_{z}^{+}<10$ in wall units. These values are based on the friction velocity from the simulation with the highest turbulence intensity.

Table 1 is an overview of the five fine-mesh simulations performed. The turbulence intensity, $T_{u}$, is defined as

$$
T_{u}=\sqrt{\frac{1}{\frac{1}{3} \frac{\overline{u^{\prime}}+\overline{v^{\prime} v^{\prime}}+\overline{w^{\prime} w^{\prime}}}{U_{o}^{2}}} .}
$$

Apart from the baseline, disturbance-free simulation T0, which is effectively twodimensional, all simulations use 129 points to resolve the span. The mean velocity contours are shown in figure 2 and correspond to the fine mesh, T0 simulation. The flow speed monotonically decreases through the passage, and the overall deceleration is $25 \%$ of the inflow velocity. The momentum change across the passage is primarily due to the reduction in the vertical ( $y$-direction) momentum. The velocity in the $x$-direction, on the other hand, is only slightly increased in order to compensate for the mass flow deficit due to the development of the boundary layers.

The surface pressure distribution, $C_{p} \equiv\left(P-P_{\text {ref }}\right) /\left(\rho U_{o}^{2} / 2\right)$, from the laminar computation on the fine mesh is shown in figure 3. The pressure surface of the blade (top curve) is subject to an APG from the leading edge till approximately $80 \%$ of the chord, after which the boundary layer is subject to FPG. A mild separation of the pressure surface boundary layer takes place at $x / L=0.365$ and extends for $24 \%$ of the 


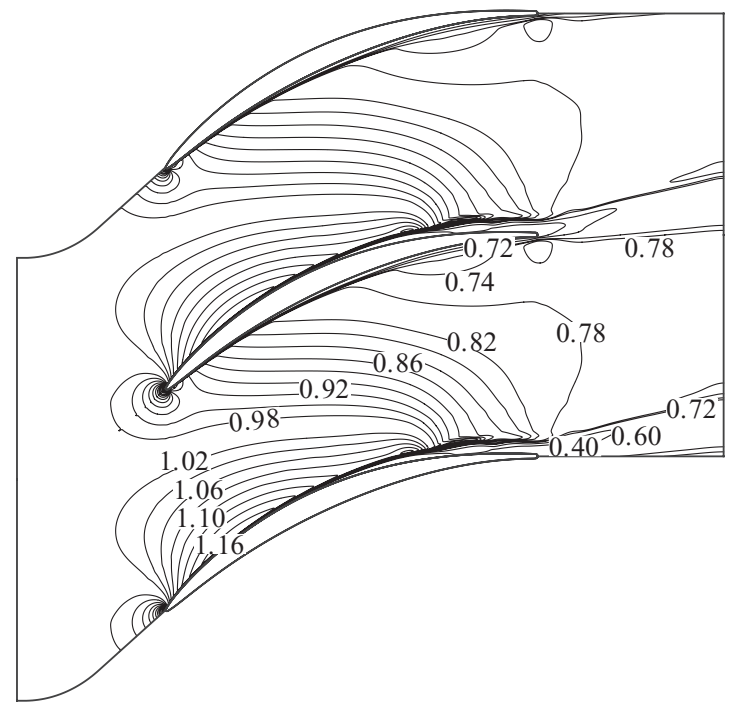

FiguRE 2. Simulation T0: contours of the time-averaged flow speed.

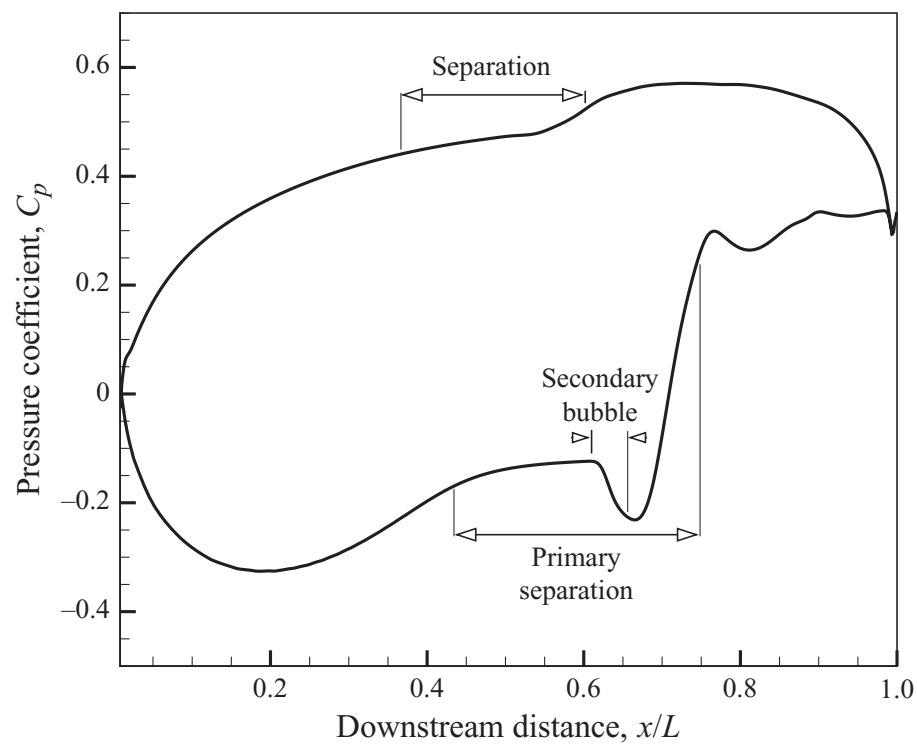

FIGURE 3. Simulation T0: pressure distribution along the blade surface. The top curve corresponds to the pressure surface and the lower curve corresponds to the suction surface.

axial chord. The suction surface (lower curve) initially undergoes strong acceleration up to $20 \%$ chord, followed by a strong APG that causes flow separation. The flow reattaches farther downstream, with separation extending over approximately $31 \%$ of the axial chord. Beyond the reattachment point, the pressure recovery resembles a wake flow. The region denoted 'secondary bubble' and the wake region of the flow, $x / L>0.8$, are only captured in the fine mesh, and would otherwise be unresolved.

Figure 4 shows the skin friction coefficient for the disturbance-free simulation, $C_{f} \equiv \mu\left(\partial u_{t} / \partial n\right) /\left(\rho U_{o}^{2} / 2\right)$, where $u_{t}$ is the velocity tangential to the blade surface, and 

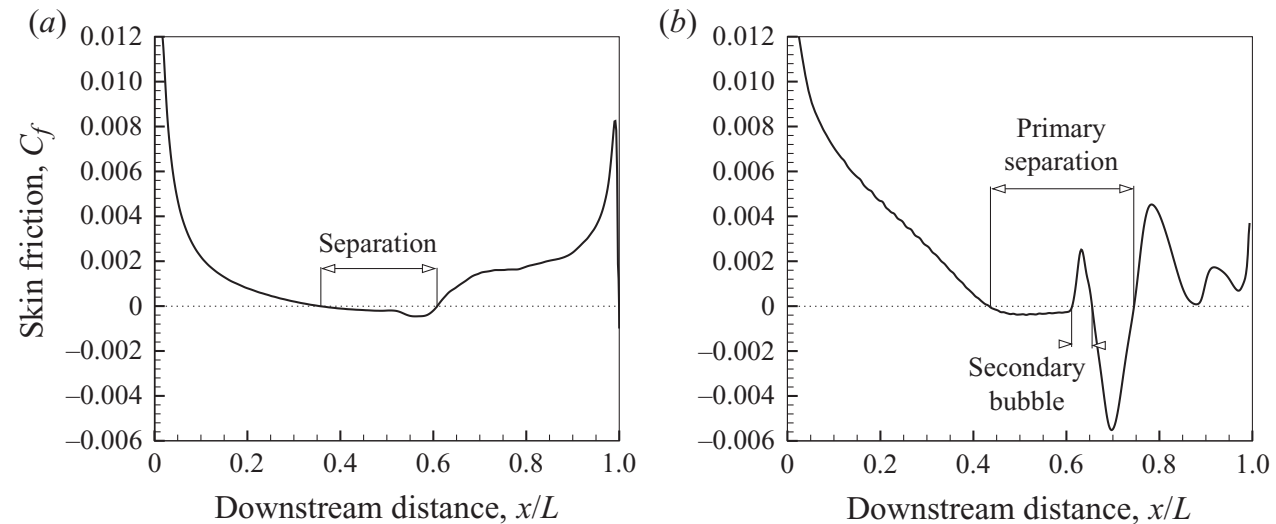

FIGURE 4. Simulation T0: skin friction on $(a)$ the pressure and $(b)$ suction surfaces of the blade. The separation regions are marked.

$n$ is the wall-normal coordinate. The separation regions on both the pressure and suction surfaces are marked. On the pressure surface, downstream of the reattachment, an increase in the skin friction is observed, which is associated with the FPG towards the trailing edge of the blade. On the suction surface, the secondary bubble is shown as a region of forward flow inside the main separation region. The skin friction increases downstream of attachment for a short region, but is subsequently reduced. The $C_{f}$ pattern is explained by considering the instantaneous flow field.

Figure 5 is an illustration of the instantaneous laminar flow around the blade surface. Contours of instantaneous vorticity capture the separated boundary layers on both sides of the blade. On the pressure surface, the separation rolls remain adjacent to the surface. The dynamics of the suction surface are more complex. The interaction of the separated $\mathrm{K}-\mathrm{H}$ rolls and the newly formed boundary layer is shown, at four phases of the shedding cycle, in figure $6(a-d)$. It is seen that the $\mathrm{K}-\mathrm{H}$ vortices convect slightly away from the wall. However, they maintain a separation region in their shadow, within the newly formed boundary layer. This interaction is similar to that reported by Jones et al. (2008). The average of the attached flow and separation in the shadow of the migrating $\mathrm{K}-\mathrm{H}$ rolls gives rise to the $C_{p}$ and $C_{f}$ patterns in figures 3 and $4(b)$.

\subsection{The free-stream turbulence}

A separate DNS of decaying, homogeneous isotropic turbulence provided the vortical inflow disturbance. This simulation was in a periodic box, whose vertical and spanwise extents exactly matched the inflow plane of the compressor domain. By Taylor's hypothesis, the streamwise coordinate of the box of turbulence can be regarded as time: thus, two-dimensional planes of $u^{\prime}, v^{\prime}, w^{\prime}$ were extracted from the turbulent box as a function of time, and superimposed onto the mean flow at $x / L=-0.4$ in the compressor DNS. It should also be noted that the viscosity of both simulations was the same, to ensure a short adjustment length for the inflow turbulence once it enters the compressor computational domain. Time averaging was carried out over approximately 18 convective time units based on the free-stream velocity and the axial chord.

Various levels of the free-stream turbulence intensity were simulated. The five cases to be considered - listed in table $1-$ are $T_{u}=\{0.00,3.25,6.5,8.0,10.0\} \%$, with 


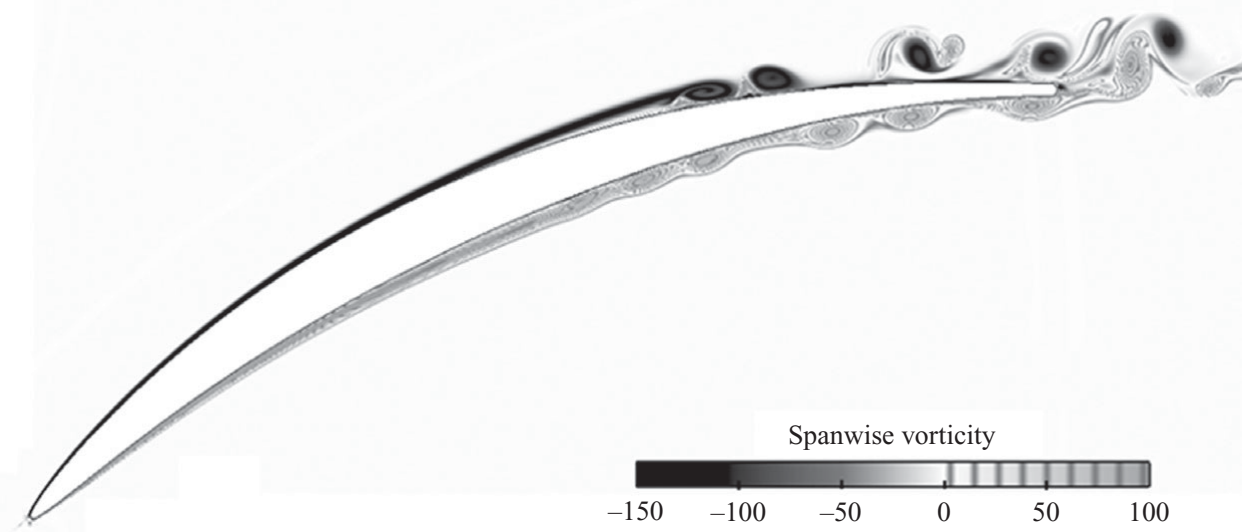

FigURE 5. Snapshot of the vorticity field in the disturbance-free simulation T0.

(a)

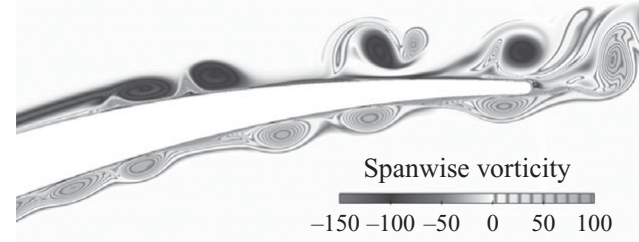

(c)

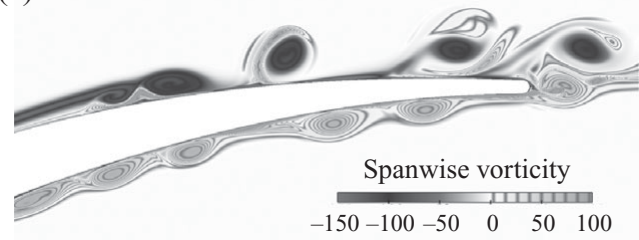

(b)

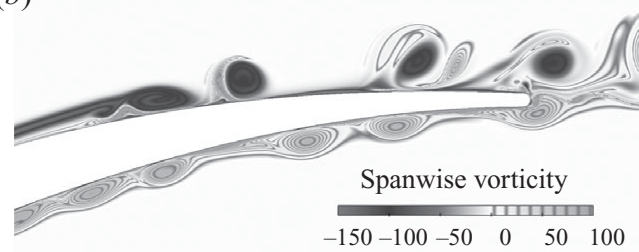

(d)

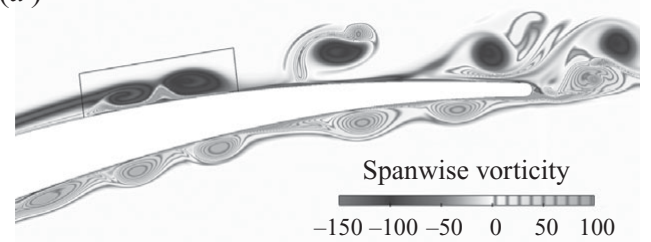

FIGURE 6. $(a-d)$ Four phases of the shedding cycle from the disturbance-free simulation T0. Total period is $T=0.24$, which corresponds to the non-dimensional frequency, $F \equiv 10^{6} \omega \nu / U_{o}^{2}=189$.

the integral length scale $L_{k}=0.06$, at the inlet plane. These highlight distinct physical phenomena. Throughout the paper, we will adhere to the following convention for graphical representation of the results: T0 (_- ); T1 (- - ); T2 ( $\left.-{ }_{-}\right)$; T3 $(-\cdots-)$; T4 ( ....).

Contours of the turbulence intensity for simulation T2 are shown in figure 7. Since the velocity ratio across the compressor is moderate (figure 2), it is expected that the mean strain would slightly reduce the energy decay rate. This reduction in the decay rate is clear in figure 7, downstream of the leading edge. Past the trailing edge, a higher decay rate is recovered.

The downstream decay of $T_{u}$ at mid-pitch is plotted in figure $8(a)$, for simulations T1-T4. The evolution of the turbulence intensity can be expressed in the form $T_{u}=A(x+B)^{C}$. Using a least-squares method, the best fit for simulation $\mathrm{T} 2$ is achieved when $C \approx-0.46$, which is weaker than the decay rate for isotropic 


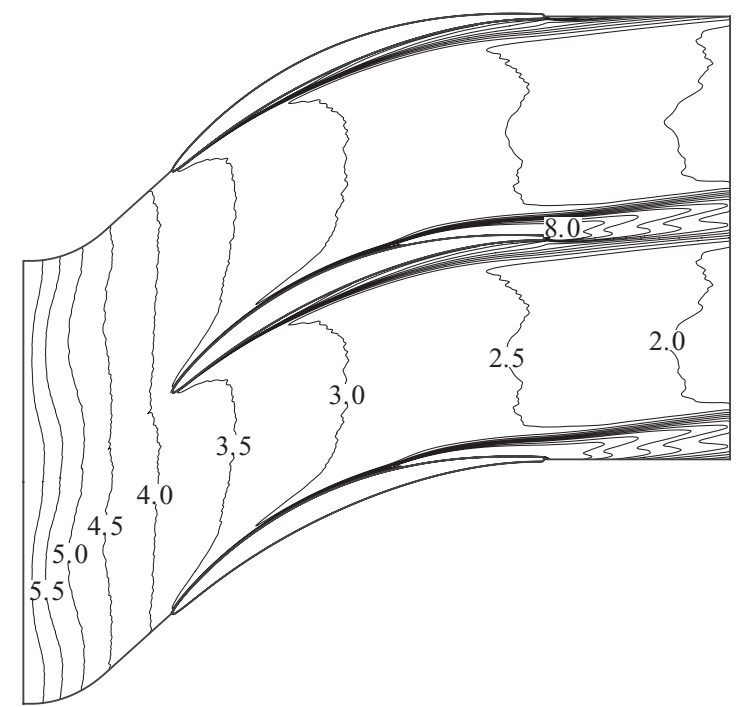

FIGURE 7. Simulation T2: contours of the turbulence intensity, $T_{u}$.

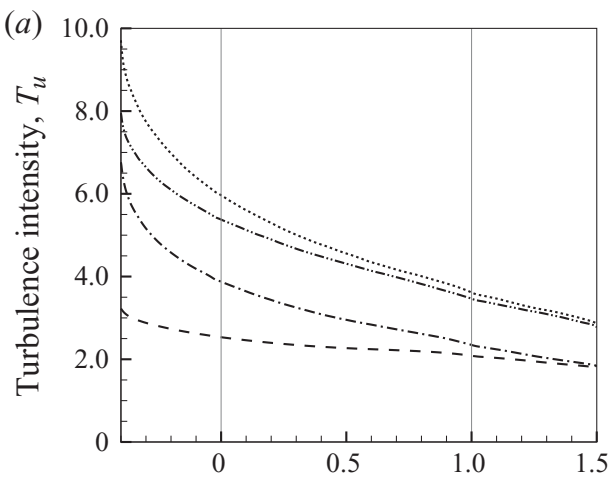

Downstream distance, $x / L$

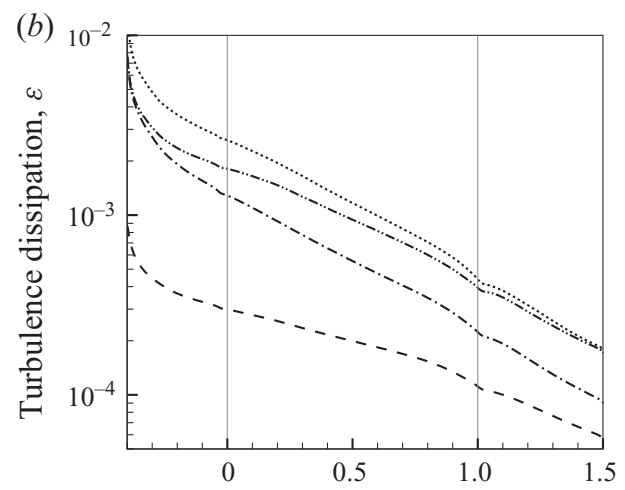

Downstream distance, $x / L$

Figure 8. Simulations T1-T4. (a) The turbulence intensity and (b) dissipation, plotted at mid-pitch from the various simulations with inflow turbulent forcing. Lines: - - $\mathrm{T} 1 ;-$ $\mathrm{T} 2 ; \ldots \ldots-\mathrm{T} 3 ; \ldots \mathrm{T} 4$.

turbulence, $C \approx-0.71$. The lower value of $|C|$ is primarily due to reduction in the turbulence decay rate within the passage. Figure $8(b)$ shows the evolution of the turbulence dissipation $\epsilon$ at mid-pitch. The dissipation was evaluated using the definition $\epsilon \equiv v \overline{\left(\partial u_{i}^{\prime} / \partial x_{j}\right)\left(\partial u_{i}^{\prime} / \partial x_{j}\right)}$.

In figure $9(a)$, the intensity at the boundary-layer edge is plotted for both the suction and pressure surfaces. Despite the pressure gradients along the blade, $T_{u}$ remains similar to the levels recorded at mid-pitch. In order to assess the isotropy of the free-stream turbulence, the fluctuation field was projected onto streamline coordinates and the root-mean-square (r.m.s.) disturbances are computed according 

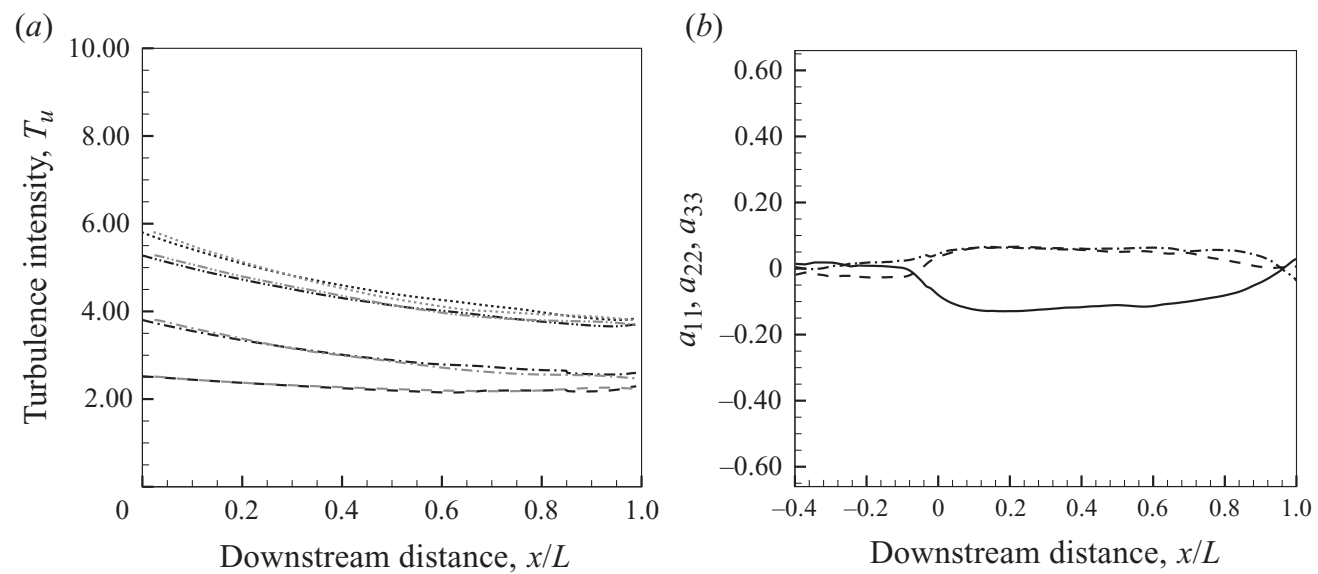

FIGURE 9. (a) The turbulence intensity at the edge of the boundary layers. Dark lines correspond to the suction surface and light lines correspond to the pressure surface. Lines: - - - T1; _ _ _ T2; _ ... - T3; .... T4. (b) Simulation T2: anisotropy of the free-stream turbulence at the edge of the suction surface boundary layer. Lines: $-a_{11} ;---a_{22}$; $--a_{33}$.

to

$$
\begin{aligned}
& u_{t}^{r m s}=\left[\overline{\left(+u^{\prime} t_{x}+v^{\prime} t_{y}\right)^{2}}\right]^{1 / 2}, \\
& u_{n}^{r m s}=\left[\overline{\left(-u^{\prime} t_{y}+v^{\prime} t_{x}\right)^{2}}\right]^{1 / 2},
\end{aligned}
$$

where $\left(t_{x}, t_{y}\right)$ is the two-dimensional, streamwise unit tangent. At mid-pitch, the turbulence remained isotropic, $u_{t}^{r m s} \approx u_{n}^{r m s} \approx w^{r m s}$, which is consistent with the lowlevel of mean strain at mid-pitch. The same was true near the pressure surface of the blade. However, the favourable pressure gradient on the suction surface caused anisotropy of the turbulence at the edge of the boundary layer. Figure $9(b)$ shows the anisotropy, $a_{i j}=\overline{u_{i}^{\prime} u_{j}^{\prime}} / k-(2 / 3) \delta_{i j}$, where the subscripts $(i, j)$ refer to the streamwise, wall-normal and spanwise directions. The results are from the intermediate turbulence intensity case T2. Upstream of the blade, the turbulence is isotropic. In the favourable pressure gradient region, $0<x / L<0.2$, the tangential component of the turbulence decays, while the normal velocity perturbations are amplified, consistent with the prediction of rapid distortion theory.

\section{The pressure surface}

The flow on the pressure surface separates in the absence of the free-stream turbulence, but remains attached when it is present. This is seen in the $C_{p}$ distribution in figure 10; it is seen more clearly in the plot of $C_{f}$, in the same figure. The slightly negative values change to positive values with free-stream turbulence.

In the presence of free-stream perturbations, even at the lowest intensity $T_{u} \sim 3.25 \%$, the boundary layer transitions to turbulence. Transition onset, defined as the point of minimum skin friction, takes place at $x / L=0.22$, which is upstream of the laminar separation point. The transitional boundary layer circumvents separation, and transition is complete at $x / L=0.46$.

Breakdown to turbulence can be caused by a wealth of mechanisms, a number of which are relevant to adverse-pressure-gradient flow on curved surfaces, and must all 


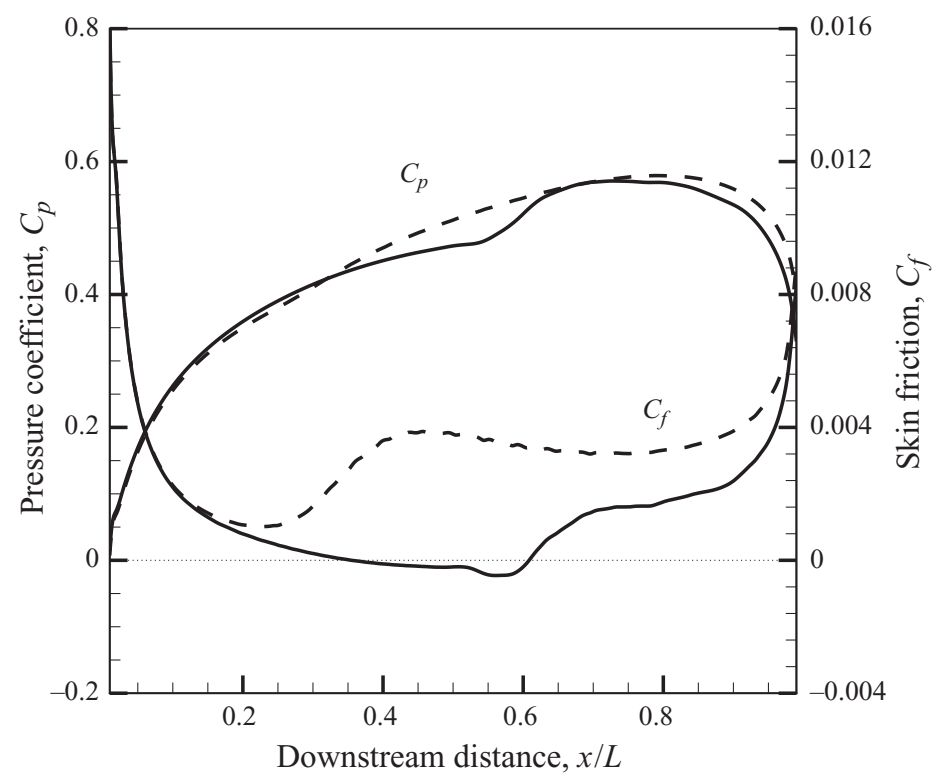

Figure 10. Pressure coefficient, $C_{p}$, and the skin friction $C_{f}$, along the pressure surface. Lines: — laminar (case T0), - - - turbulent (case T1).

be considered. First, the interaction of the separated shear layer with the free-stream turbulence can lead to a turbulent boundary layer. Here, $\mathrm{K}-\mathrm{H}$-type instabilities are most relevant. However, if transition takes place upstream of the separation point, bypass transition is suspect (Klebanoff et al. 1962; Morkovin 1969; Westin et al. 1994). Free-stream vortical forcing can lead to the generation of algebraically amplifying Klebanoff distortions, which become the seat for secondary instability and breakdown to turbulence (Jacobs \& Durbin 2000). On a concave wall, the Klebanoff modes can give way to exponentially amplifying Görtler vortices (Görtler 1940).

It should be noted, however, that bypass breakdown is not inevitable in response to the free-stream turbulence. The presence of a leading edge and adverse gradient in the mean pressure can cause $\mathrm{T}-\mathrm{S}$ waves to amplify quickly, and to dominate, or contribute to, the transition process. Hughes \& Walker (2001) for instance asserted, based on hotwire measurements of transitional boundary layers in an adverse pressure gradient, that high-frequency velocity oscillations could be related to discrete instability waves. Added complexity arises since both the $\mathrm{T}-\mathrm{S}$ waves and their secondary instability are known to be influenced by the disturbance environment. In a streaky boundary layer, the T-S wave growth rate can be altered (Cossu \& Brandt 2002; Fransson et al. 2005), and its secondary instability can change appearance, or wavenumber, as well as growth rate (Liu et al. 2008a,b).

\subsection{Averaged results}

In order to determine whether a Görtler instability plays a role, the Görtler number, $G \equiv\left(\bar{u}_{t}^{\infty} \theta / v\right)(\theta / \mathscr{R})^{1 / 2}$ is plotted versus downstream distance in figure 11 . Here, $\bar{u}_{t}^{\infty}$ is the mean streamwise velocity at the edge of the boundary layer, $\theta$ is the momentum thickness of the boundary layer, $v$ is the kinematic viscosity and $\mathscr{R}$ is the radius of curvature of the wall. The lines extend up to the location where separation or transition to turbulence is observed. According to the previous studies of the Görtler instability (Saric 1994), the boundary layer flow on a concave surface becomes unstable at 


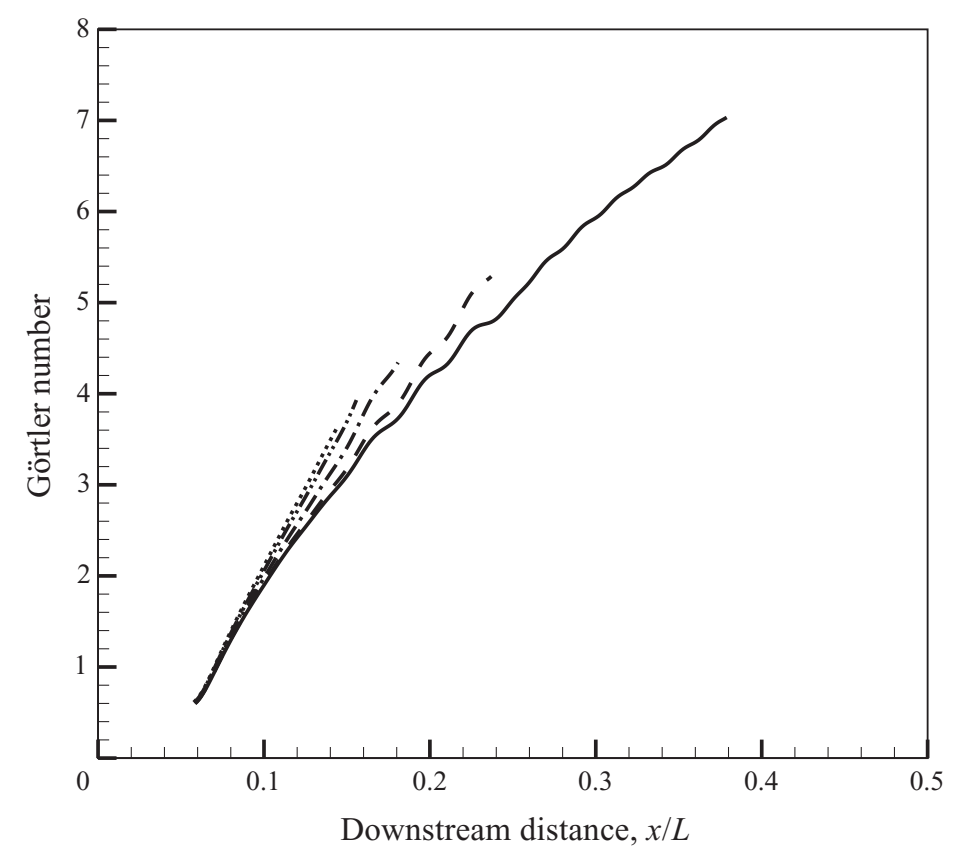

FIGURE 11. Simulations T0-T4: Görtler number on the pressure surface, upstream of transition or separation, $G \equiv\left(\bar{u}_{t}^{\infty} \theta / \nu\right)(\theta / \mathscr{R})^{1 / 2}$. Lines: — $\mathrm{T} 0 ;--$ - T1; — - — T2; $-\cdots-\mathrm{T} 3 ; \ldots \mathrm{T} 4$.

$G \sim 0.3$ and the vortices are detected when $G \sim 5-6$; secondary instability is observed for $G \sim 6-9$. As seen in figure 11, only in the laminar simulation T0 does a significant streamwise interval $(\Delta x=0.15 L)$ exist where $G>5$. However, in our simulations the instability was not observed. In all other simulations, an interval where $G>5$ does not exist or is too short for Görtler vortices to be detected. Indeed, vortex-identification methods did not yield any evidence of Görtler vortices on the aerofoil surface. Both the $Q$ and $\lambda_{2}$ criteria (Jeong \& Hussain 1995) were considered. The absence of Görtler vortices was independent of the spanwise size of the domain. Here, the ratio of the spanwise extent to the maximum shear-layer thickness is approximately 10 , similar to the DNS of Jones et al. (2008) of separation bubbles on the NACA-0012 aerofoil.

For simulation T1 all three components of the r.m.s. disturbance profile, upstream of transition, are shown in figure 12. The streamwise perturbation velocity reaches the highest amplitude and resembles Klebanoff distortions which precede bypass transition to turbulence. The wall-normal location of the maximum tangential velocity disturbance is shown by a circle in the figure. The maximum $u_{t}^{r m s}$ moves away from the wall with downstream distance. As a result, the wall-normal perturbation, $u_{n}^{r m s}$, increases at that location as well (see a zoomed-in view of $u_{n}^{r m s}$ in figure 13).

For simulations T1-T4, the maximum $u_{t}^{r m s}$ is plotted versus downstream distance in figure 14(a). The associated $u_{n}^{r m s}$ at the same location is also shown. The increase in the wall-normal disturbance may be due to curvature effects. In figure $14(b)$, the $u_{n}^{r m s}$ disturbance from simulations T1-T4 is shown at a constant height from the wall. The amplification due to curvature is still observed, but takes place farther downstream and is less pronounced than in figure 14(a). These observations are consistent with the absence of Görtler vortices on the pressure surface upstream of transition. 
(a)

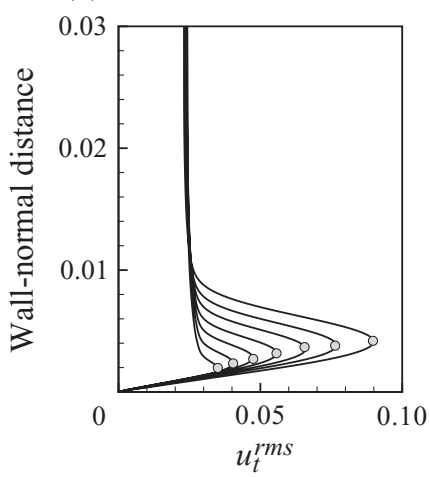

(b)

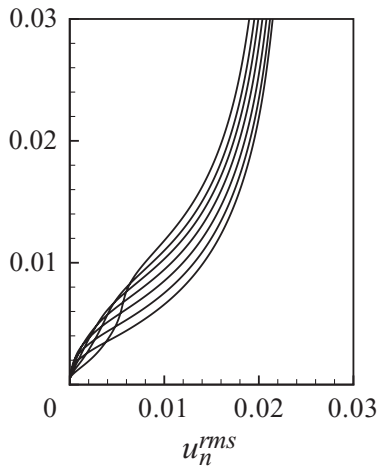

(c)

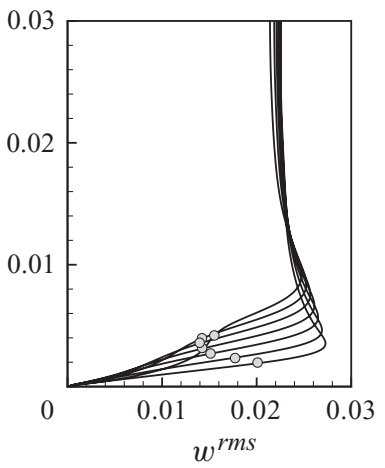

Figure 12. Simulation T1. $(a-c)$ Profile of the r.m.s. perturbation inside the boundary layer, upstream of transition, $x / L=\{5,10,15,20\} \times 10^{-2}$. The circles mark the wall-normal locations where $u_{t}^{r m s}$ reaches its maximum value.

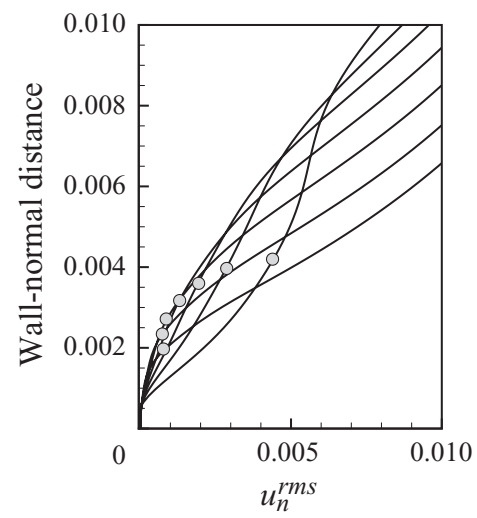

FIGURE 13. Simulation T1: a zoomed-in view of $u_{n}^{r m s}$ from figure 12, inside the boundary layer. The profiles are extracted upstream of transition at $x / L=\{5,10,15,20\} \times 10^{-2}$.

(a)

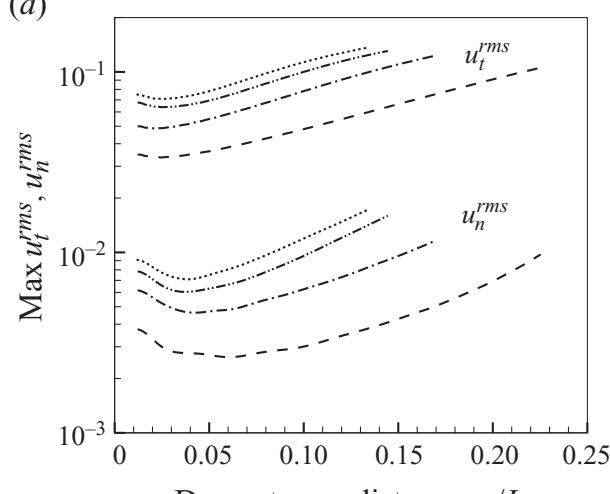

Downstream distance, $x / L$ (b)

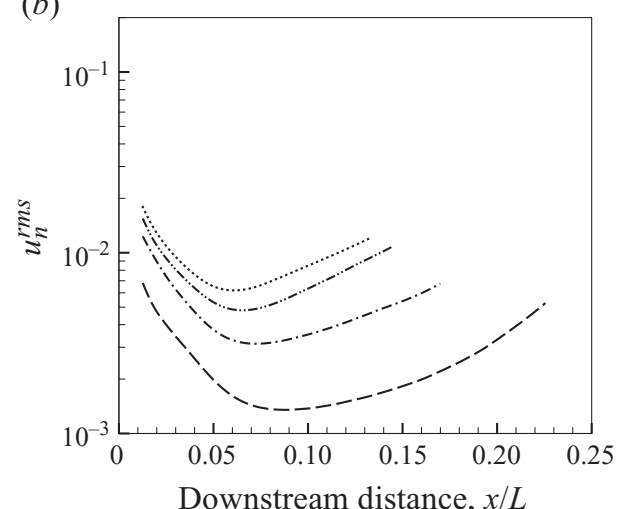

FIgURE 14. Simulations T1-T4. (a) The maximum $u_{t}^{r m s}$ and the associated $u_{n}^{r m s}$ versus downstream distance. $(b)$ The r.m.s. normal perturbation $u_{n}^{r m s}$ at a fixed distance, $d=0.002$, from the pressure surface. Lines: - - T1; - - — T2; — - - T3; -.. T4. 


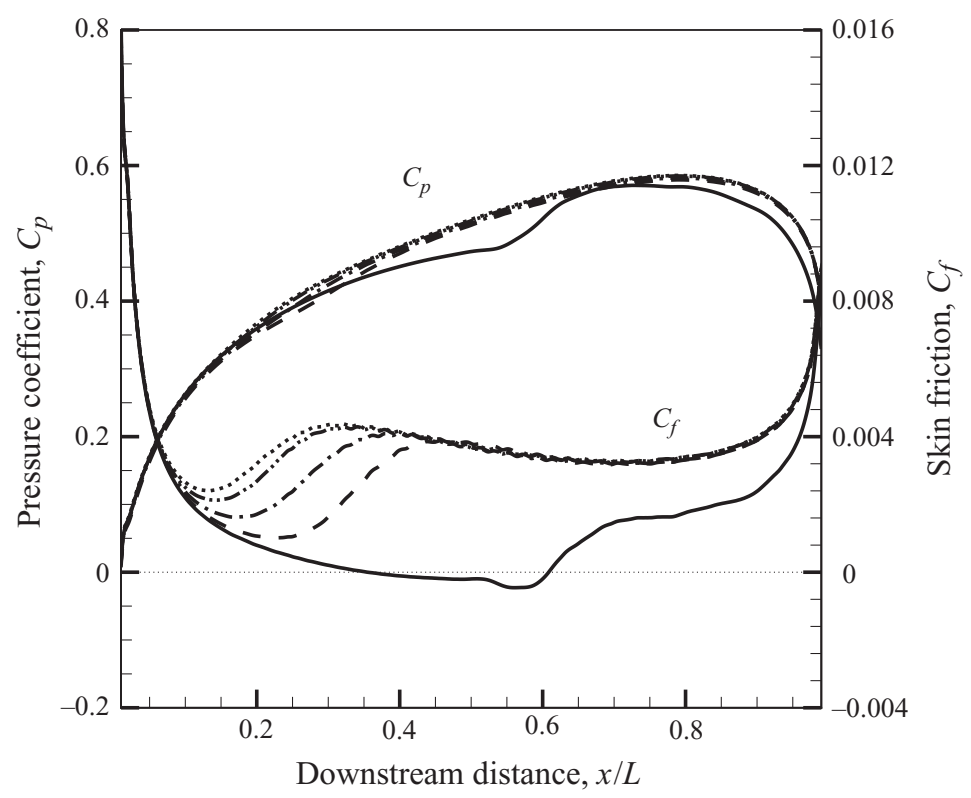

Figure 15. Pressure coefficient, $C_{p}$, and the skin friction $C_{f}$, along the pressure surface. Lines: — $\mathrm{T} 0 ;-\ldots \mathrm{T} 1 ; \_-\ldots \mathrm{T} 2 ; \_\ldots-\mathrm{T} 3 ; \ldots \mathrm{T} 4$.

As the free-stream turbulence intensity is increased, the amplitude of the Klebanoff modes also increases. As a result, their secondary instability and breakdown to turbulence are promoted (Andersson et al. 1999). The upstream shift in the transition onset at higher $T_{u}$ is well documented in the literature, and is captured in figure 15, where both the pressure coefficient and the skin friction are plotted for simulations T1-T4. Transition location is observed to move upstream monotonically as the turbulence intensity is increased. In addition, the sensitivity of the transition onset to $T_{u}$ is reduced at higher free-stream turbulence levels, consistent with the data correlation of Abu-Ghannam \& Shaw (1980).

The downstream evolution of the momentum thickness Reynolds number and the boundary-layer thickness, upstream of transition, is shown in figure 16. Initially both quantities are equal in all simulations, but deviate downstream for different freestream conditions. Prior to transition, the deviation is due to mean flow distortion by Klebanoff modes.

Despite the required long integration time, frequency spectra were computed,

$$
E(x, y, \omega)=\int_{-\infty}^{+\infty}\langle u(t) u(t+\tau)\rangle \mathrm{e}^{\mathrm{i} \omega \tau} \mathrm{d} \tau .
$$

The angle brackets $\langle\cdot\rangle$ denote averaging in the spanwise direction. The highest resolvable frequency, $F_{\max }=2.2 \times 10^{5}$, is determined by the Nyquist criterion for the size of the computational time step. The lower frequency limit, on the other hand, is determined by the integration period for each simulation, which was $T=18.15 L / U_{\infty}$. The corresponding minimum non-dimensional frequency is therefore $F_{\min } \equiv 10^{6} \omega \mathrm{v} / U_{o}^{2}=2.5$. For comparison, the lowest frequency in the inflow turbulence is $\min \left\{F_{\text {inlet }}\right\}=168$. Therefore, the integration time spans 67.4 sweeps of the inflow turbulence box. 
(a)

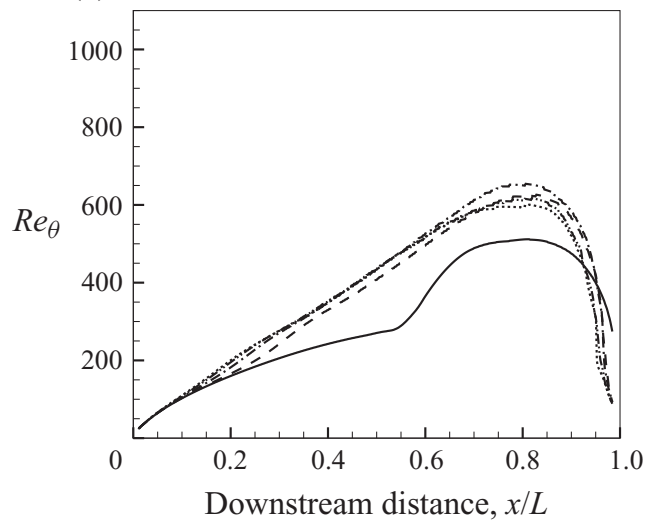

(b)

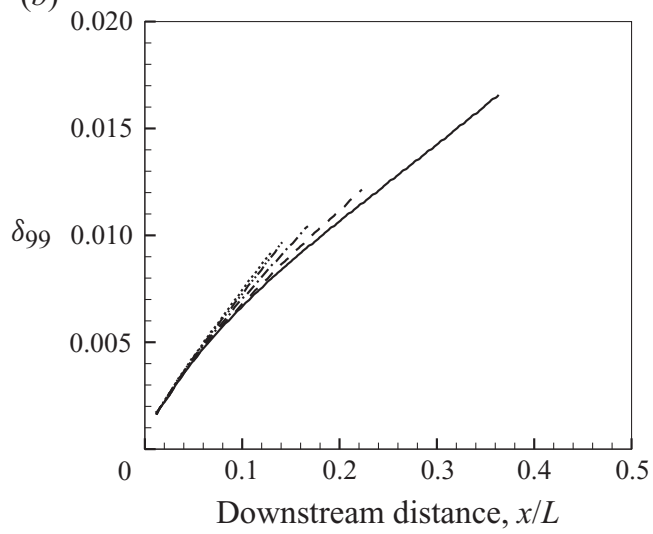

Figure 16. Simulations T0-T4. (a) Downstream variation of the momentum thickness Reynolds number $\operatorname{Re}_{\theta}$ and $(b)$ the boundary layer thickness upstream of transition. Lines: - T0; - - T1; — - — T2; — . - T3; ... T4.
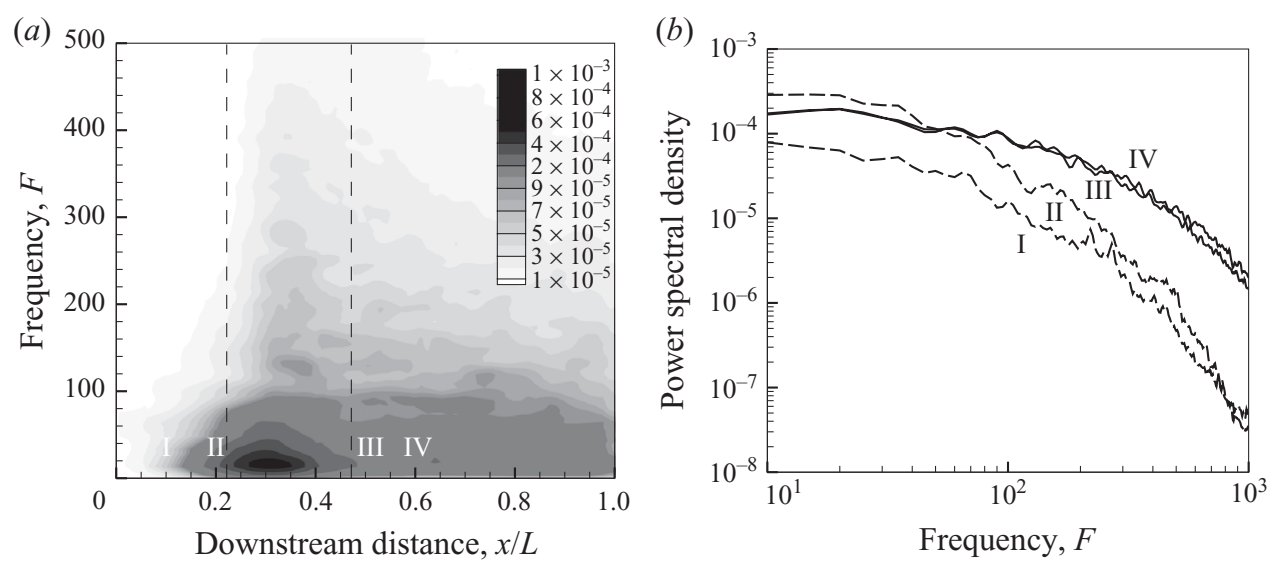

FIGURE 17. Simulation T1: frequency spectra of the streamwise velocity perturbation, at the wall-normal location where the disturbance energy is maximum. $(a)$ Contours of the power spectral density are plotted versus downstream distance and frequency. The dashed lines mark the onset and end locations of transition to turbulence. $(b)$ Line plots of the power spectral density versus frequency at locations I-IV, which correspond to $x / L=\{0.1,0.2,0.4,0.5\}$, respectively.

The streamwise evolution of the energy spectra for simulation T1 is shown in figure 17. At each downstream location, the wall-normal location where $u_{t}^{r m s}$ is maximum is selected for the evaluation of the energy spectral density. It is clear from figure $17(a)$ that upstream of transition, the disturbance inside the boundary layer is dominated by a low-frequency component. This is consistent with previous observations of Jacobs \& Durbin (2000). The highest energy levels occur in the frequency range $15<F<35$, which is significantly lower than the frequencies of the turbulence introduced at the inlet plane, $\min \left\{F_{\text {inlet }}\right\}=168$. The generation of Klebanoff modes must, therefore, be preceded by nonlinear interactions, which generate low-frequency disturbances. Only these low-frequency perturbations penetrate the boundary layer shear, and are amplified by the lift-up mechanism. Therefore, they 
become dominant in the near-wall region. Beyond the transition region, the flow becomes fully turbulent and the spectrum is broadband.

The change in the disturbance spectra from the pre-transitional to the turbulent region is demonstrated in figure 17(b). In the pre-transitional region, proceeding from locations I to II, energy accumulates mainly in the low frequencies. At locations III and IV, which are both in the fully turbulent regime, the energy has filled in at higher frequencies.

Thus far, only averaged flow quantities were considered, and have all provided clear evidence of bypass transition. Next, the instantaneous fields from simulations of various free-stream conditions are presented and provide a new perspective on the nature of the boundary-layer breakdown in these simulations.

\subsection{Instantaneous results}

An inspection of the instantaneous fields from the simulations of various turbulence intensities demonstrates that transition on the pressure surface can take place via three different mechanisms. The dominant route to boundary-layer turbulence is a function not only of the free-stream intensity but also of the flow condition within the boundary layer. First, the discussion will focus on the lowest turbulence intensity simulation T1, followed by the higher $T_{u}$ cases.

\subsubsection{The inner mode}

The first transition mechanism observed on the pressure surface boundary layer is shown in figures 18-20. Figure 18 is a plane view of the tangential velocity perturbation field inside the boundary layer, at a distance $d \sim 1.64 \times 10^{-3}$ from the wall. The sequence of images corresponds to a time period $\Delta t=0.2$. The perturbation field captures the streaky, laminar boundary layer, the transition region, and the downstream, fully turbulent flow. The streaks or Klebanoff distortions (Wu 2001) have traditionally been regarded as a precursor of bypass transition. In the current case, however, the nature of the breakdown is not evident. The middle time instance $t=0.10$ has superimposed an iso-surface of the second eigenvalue of the rate of strain tensor, $\lambda_{2}=-150$. The iso-surface highlights the presence of strong vortices, which take the shape of the letter $\Lambda$. The presence of $\Lambda \mathrm{s}$ suggests a natural transition mechanism. However, they are not independent of the streaks, or else they would be observed homogeneously across the spanwise extent of the domain. Instead, they appear in localized regions of the flow. Their spanwise size is not directly related to the streaks: these wide $\Lambda$-structures straddle more than one spanwise wavelength. They are 'lifted' away from the wall, which is clear from the figure since they lie above the plane of the tangential velocity perturbation.

The nature of the breakdown mechanism is more evident when the wall-normal perturbation field is considered (figure 19). Contours of $u_{n}^{\prime}$ perturbation amplify downstream as the $\Lambda$-shaped disturbance is convected. The $\Lambda$ structures finally break down to turbulence, sustaining the fully turbulent boundary layer farther downstream.

The designation 'inner mode' derives from the shape of the disturbance, which is clearly seen in a side view of the perturbation field (figure 20). The streamwise velocity disturbance is plotted in a plane which bisects $\Lambda \mathrm{s}$, at $t=-0.05$ relative to the time sequence in figures 18 and 19. The light and dark contours correspond to positive and negative velocity perturbations, respectively. Figure 20(a) shows that the $\Lambda$-structures are initiated adjacent to the wall, below the long forward-velocity region. Their near-wall peak is consistent with the classical T-S instability for which the critical layer is close to the wall. The wall-normal profile of the perturbation at the location of the dashed line is shown in figure 20(b). 
(a)

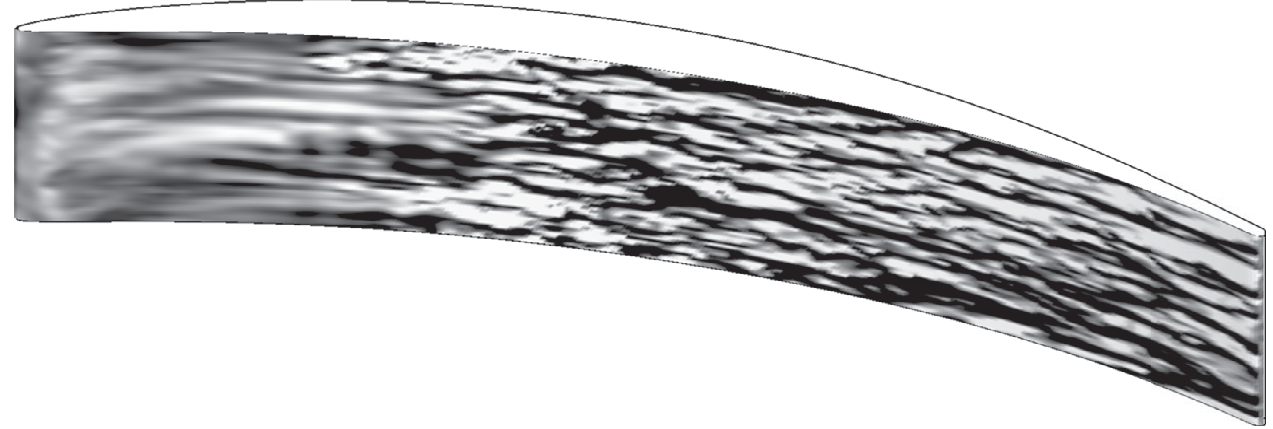

(b)

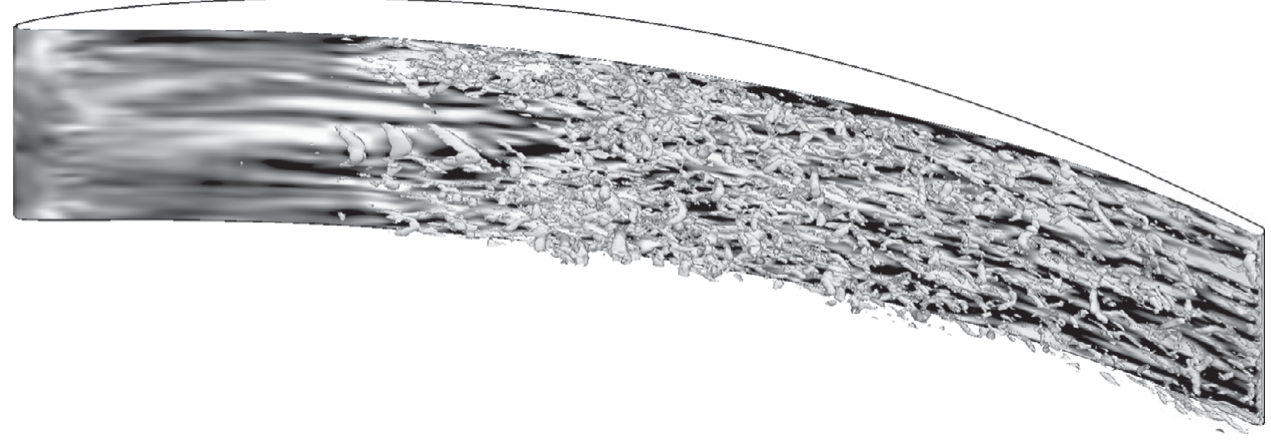

(c)

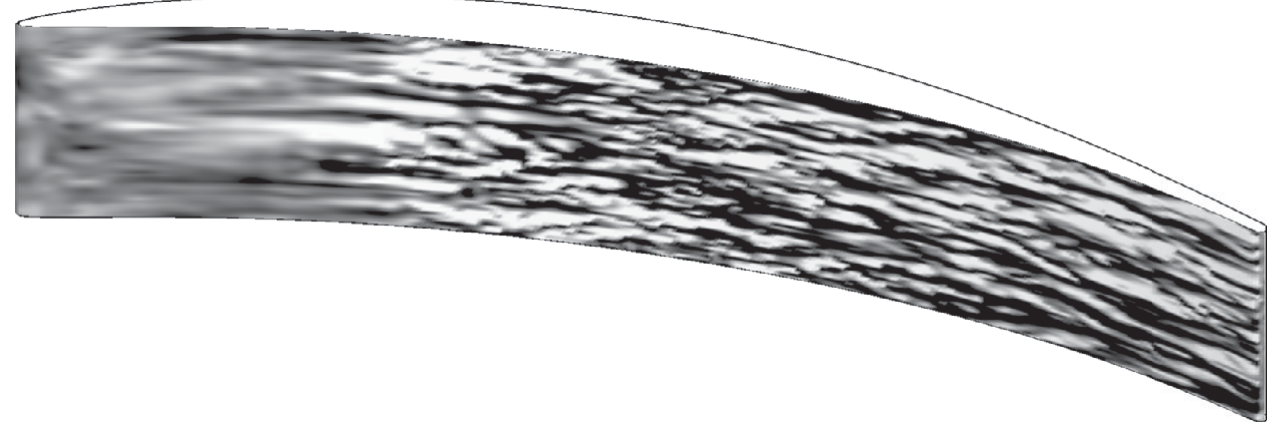

FiguRE 18. Simulation T1. $(a-c)$ Contours of the tangential velocity perturbations, $-0.1<u_{t}^{\prime}<0.1$. The plane shown is inside the pressure surface boundary layer, at a distance $d \sim 1.64 \times 10^{-3}$ from the wall. The sequence spans a time period $\Delta t=0.2$. The second time instance is included with an iso-surface of $\lambda_{2}$ vortex-identification criterion superimposed on the perturbation field.

The streamwise wavenumber of the inner instability mode is of the order of $\alpha=125$, and its non-dimensional frequency is $F=330$. Therefore, the computed phase speed, $c=0.36$, is commensurate with linear theory for discrete instability waves.

\subsubsection{The overlap mode}

The second transition mechanism observed on the pressure surface boundary layer is also an inner instability, and has its origin in the discrete linear modes, or $\mathrm{T}-\mathrm{S}$ waves. It originates near the upstream edge of the long, low-speed perturbation region, where it overlaps with the downstream edge of the high-speed region. 
(a)
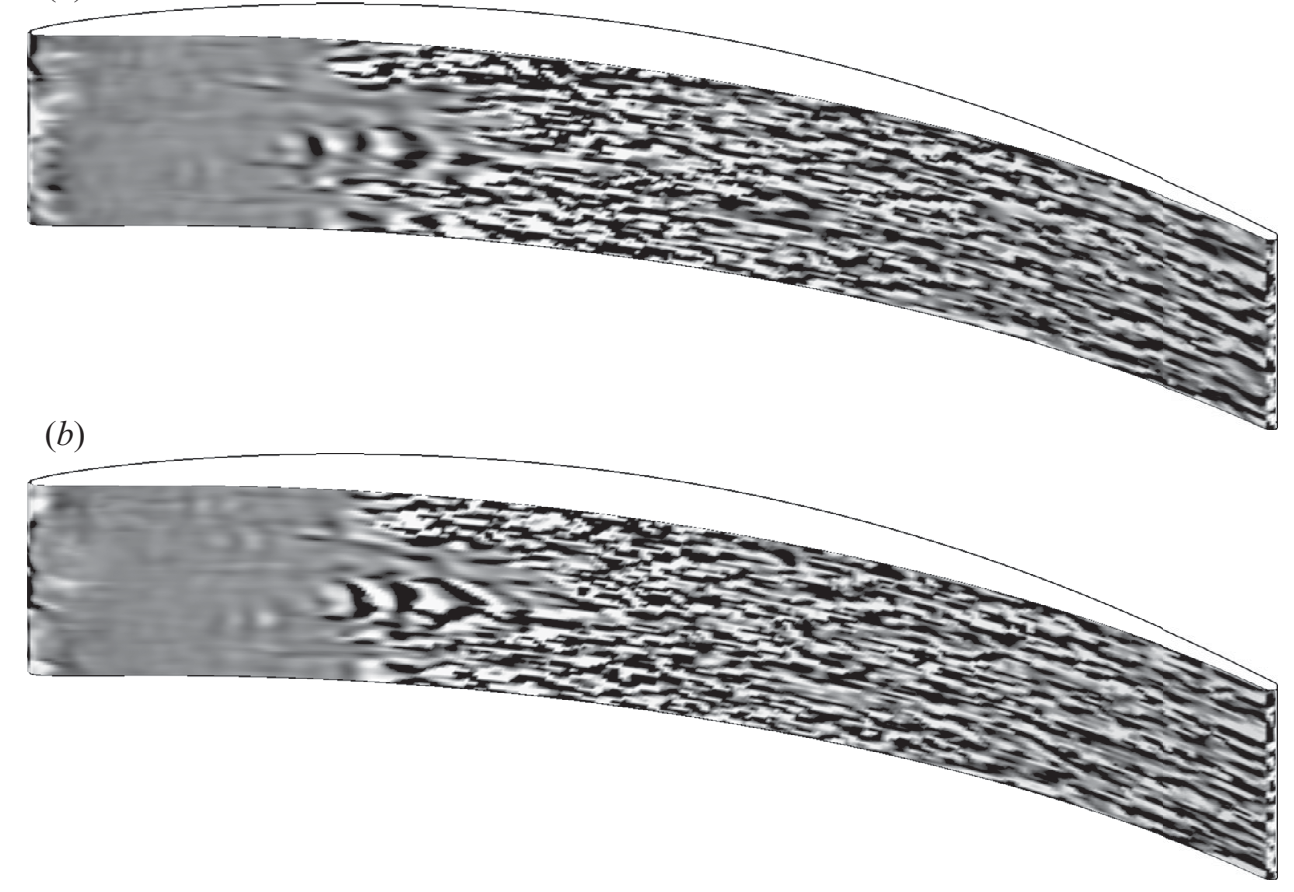

(c)

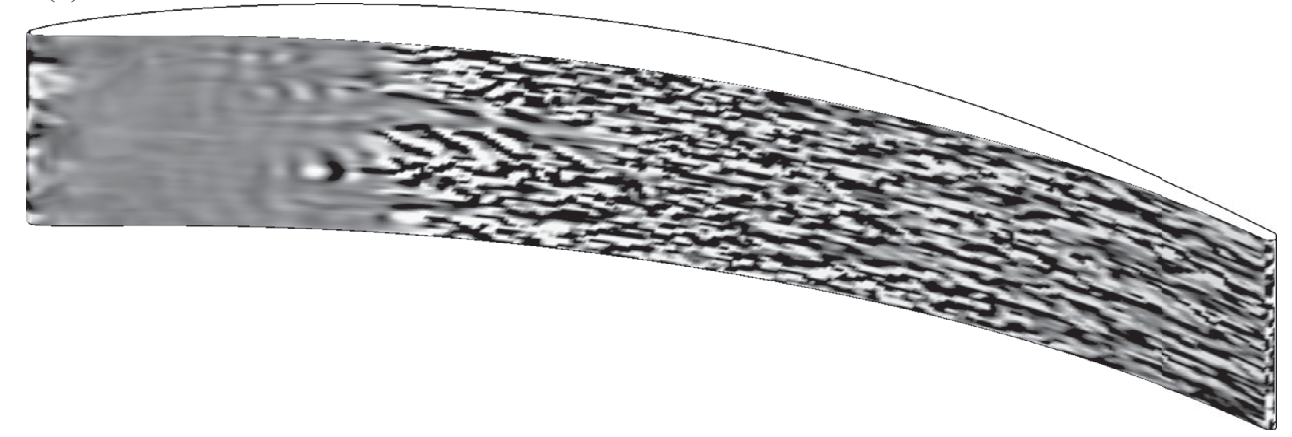

FIgURE 19. Simulation T1. $(a-c)$ Contours of the normal velocity perturbations $-0.01<u_{n}^{\prime}<0.01$. The sequence is at the same times as in figure 18 .

The initial amplification of this mode is shown in figures 21 and 22. Again, the perturbation field is plotted in a plane located at a distance $d \sim 1.64 \times 10^{-3}$ away from the blade surface; the sequence of images also corresponds to a time period $\Delta t=0.2$. The tangential velocity field is dominated by high-amplitude perturbations, which obscure the instability mechanism. It is more revealing to consider the wallnormal disturbance, which is shown in figure 21. The instability wave is marked in the first frame, and amplifies at subsequent times. The second time instance, $t=0.1$, also shows the iso-surface $\lambda_{2}=-150$. The observed vortex structures associated with the instability are narrow in the spanwise extent, in contrast to the wider $\Lambda$ s discussed above. Here, the instability has the same size as the host streak. The streamwise wavenumber and frequency are only slightly increased: the former is of the order 
(a)

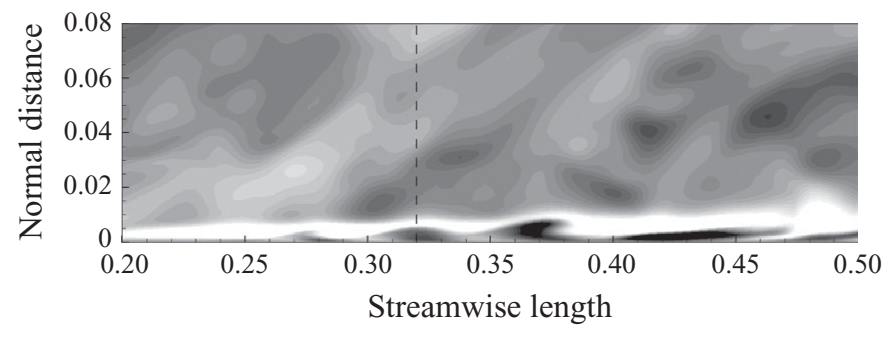

(b)

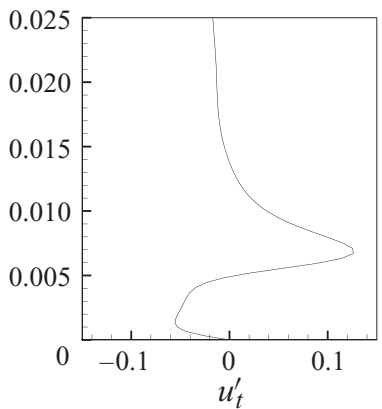

Figure 20. Simulation T1. (a) Side view of the tangential perturbation $-0.1<u_{t}^{\prime}<0.1$, which precedes the formation of the $\Lambda$-structures in figures 18 and 19 . The snapshot corresponds to $t=-0.05$, relative to the first frame in those figures. $(b)$ Wall-normal profile of the tangential perturbation extracted at the location of the dashed line.

of $\alpha=180$, and its non-dimensional frequency is $F=460$. Therefore, the computed phase speed, $c=0.36$, remains commensurate with linear, discrete instability waves.

The intermediate snapshot of figure 21, corresponding to $t=0.1$, is further inspected in figure 22. Both a plane and a side view of the tangential velocity perturbations are shown. The former shows the elongated boundary layer disturbance, which hosts the instability. The dashed line marks the location of the side view, as shown in figure $22(b)$. The side view bisects the disturbance and clearly shows the instability pattern near the upstream edge of the low-speed (dark) region.

Established literature suggests that the bypass mechanism dominates boundarylayer transition at turbulence intensities greater than $1 \%$. However, the instability modes observed on the pressure surface thus far do not resemble the instability on lifted, backward jets, observed in the previous DNS of bypass transition (e.g. Jacobs \& Durbin 2000; Nagarajan et al. 2007). Instead, they are near-wall modes and travel at the phase speed of the boundary layer's natural, discrete instability waves. This does not preclude, however, that they are influenced by the presence of streaks. Previous work by Cossu \& Brandt (2002) and Liu et al. (2008b) demonstrated that the growth rate of discrete instability waves is affected by the presence of Klebanoff distortions. In addition, Liu et al. (2008a) have shown that the secondary instability and spanwise size of the emergent $\Lambda$-structures is a function of the their spanwise wavelength. However, the mechanism is not a streak instability, rather it originates in the convection of $\mathrm{T}-\mathrm{S}$ waves by the strong, forward and backward jets of the Klebanoff distortion.

\subsubsection{Bypass transition}

The above discussion focused on the instability waves observed when the free-stream turbulence intensity at the inlet is $3.25 \%$. As the intensity is increased to $6.5 \%$, the above mechanisms are still observed. However, they are also supplemented by the bypass transition mechanism which Jacobs \& Durbin (2000) and Wu et al. (1999) describe as a secondary instability of lifted low-speed shear layers when they reach the top of the boundary layer, and are perturbed by the free-stream high-frequency eddies. The lifted shear layers have the appearance of backward jets, in the sense that the profile of the perturbation velocity is that of a jet opposite to the mean flow direction. 
(a)
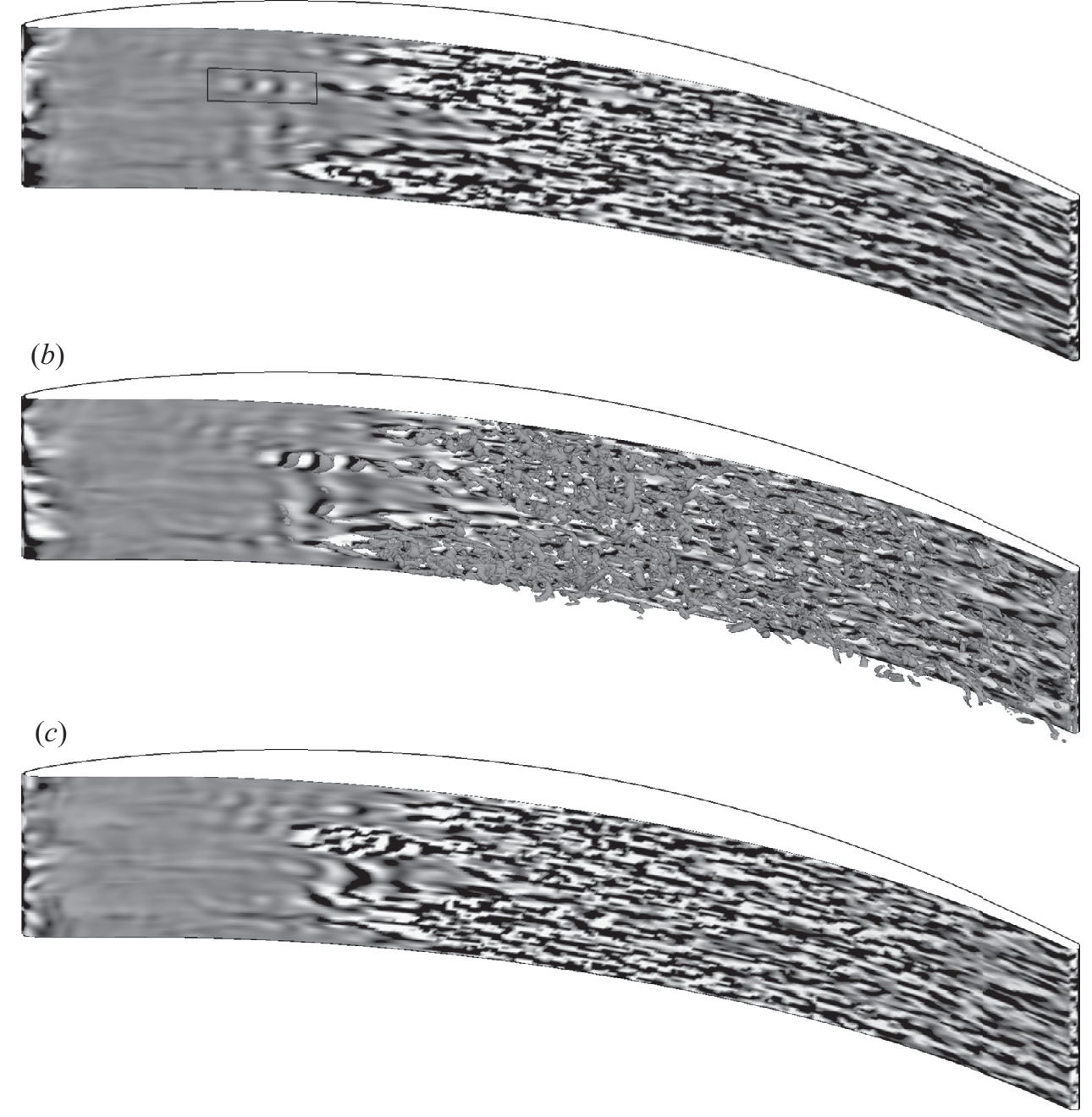

FIGURE 21. Simulation T1. $(a-c)$ Contours of the normal velocity perturbations, $-0.01<u_{n}^{\prime}<0.01$. The plane shown is inside the pressure surface boundary layer, at a distance $d \sim 1.64 \times 10^{-3}$ from the wall. The second time instance is included with an iso-surface of $\lambda_{2}$ superimposed on the perturbation field. The sequence spans a time period $\Delta t=0.2$.

For simulation $\mathrm{T} 2$, the tangential velocity perturbations inside the boundary layer do not differ qualitatively from T1: they are highly elongated in the flow direction. However, they reach higher amplitudes, of the order of $15 \%$ of the free-stream speed. The tangential perturbations preserve their elongated appearance through the transition length and into the fully turbulent boundary layer. They, thus, do not provide a clear distinction between the laminar, transitional and turbulent regions of the flow. Instead, the transition process is best identified using the wallnormal or spanwise components of the fluctuation. Figure 23 shows the perturbation field at three times. The sequence spans a period $\Delta t=0.24$ convective time units. Figure 23(a,b) shows the wall-normal perturbation and figure 23(c) shows the spanwise disturbance. The laminar, transitional and turbulent boundary layers are 
(a)

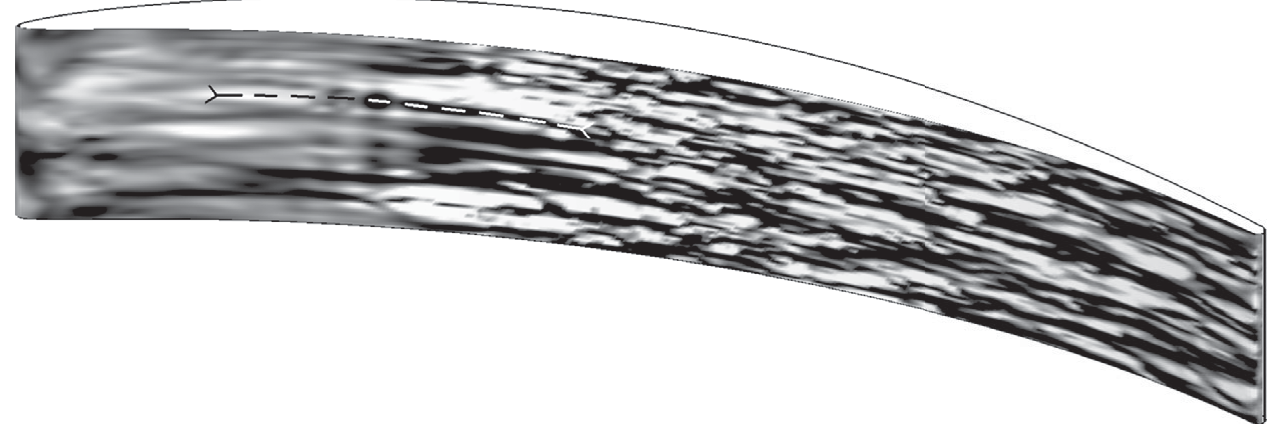

(b)

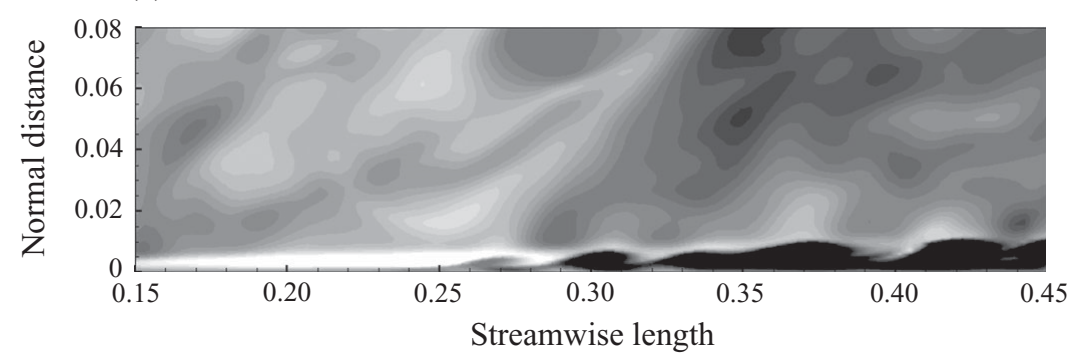

Figure 22. Simulation T1. $(a)$ Plane and $(b)$ side views of the tangential perturbation field, $-0.1<u_{t}^{\prime}<0.1$, at the intermediate time in figure 21 . The plane view shows that the instability occurs near the upstream edge of the low-speed streak and the downstream edge of the high-speed streak. The dashed line marks the location of the side view $(b)$. The side-view plane bisects the disturbance wave which leads to breakdown.

distinctly identified in figure 23. In addition, the mean locations of the onset, $X_{s}$, and end, $X_{e}$, of the transition length are shown in the figure. Upstream of $X_{s}$, the boundary layer is insignificantly perturbed. In the region $X_{s}<X<X_{e}$, a representative turbulent spot is enclosed by a rectangle in the figure. For $X>X_{e}$, the spots have spread and merged together, thus forming a fully turbulent boundary layer.

Jacobs \& Durbin (2000) suggested that breakdown in bypass transition commences near the top of the boundary layer, when the negative $u_{t}^{\prime}$ perturbations are exposed to high-frequency forcing in the free stream. This breakdown mechanism was also verified by Zaki \& Durbin (2005) in their simulations of continuous mode transition. In the current DNS, events leading to the formation of the turbulent spot in figure 23 were inspected in order to identify whether a similar mechanism takes place. Figure 24 shows profiles of the velocity perturbation vectors at the first two time instants of figure 23. The plane shown bisects the location of the spot inception. The profiles show that the perturbation vectors are pointed upstream and have the appearance of backward jets. The perturbation lifts towards the top of the boundary layer, where it is exposed to the free-stream turbulence. The interaction, which can be seen at the far left in figure $24(b)$, is destabilizing and initiates breakdown to turbulence. Therefore, despite the pressure gradient and the presence of a leading edge, the observed breakdown on the pressure surface is similar to bypass transition in flatplate boundary layers. 
(a)
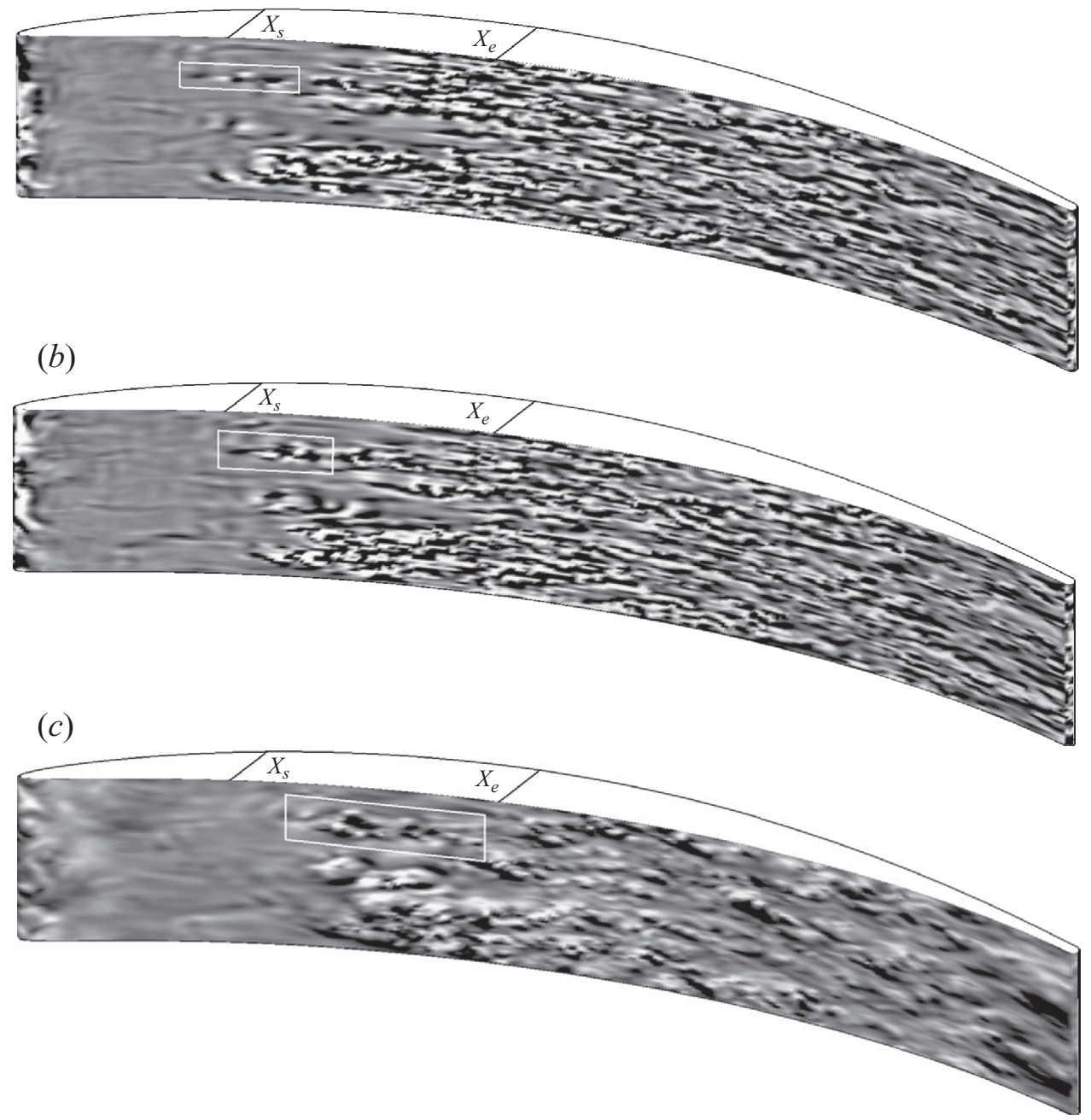

Figure 23. Simulation T2. (a,b) Contours of the wall-normal $-0.01<u_{n}^{\prime}<0.01$ and (c) spanwise $-0.1<w^{\prime}<0.1$ velocity perturbations. The time sequence spans $\Delta t=0.24$ time units.

\section{The suction surface}

In this section, the flow on the suction surface under the different free-stream turbulence levels is discussed. The focus is initially on simulations T1 and T2, which are compared to the quiescent free-stream condition and the pressure surface. This is followed by a discussion of the effect of higher $T_{u}$ on the suction surface boundary layer, using the results from $\mathrm{T} 3$ and $\mathrm{T} 4$.

\subsection{The influence of moderate free-stream turbulence intensity ( $T 1, T 2)$}

The pressure coefficient, $C_{p}$, of the full blade is shown in figure 25(a), for cases T0, T1 and T2. Unlike the pressure surface, where the flow is attached in the presence of the free-stream turbulence, the suction surface boundary layer remains separated independent of the free-stream condition, be it quiescent or turbulent. The persistence of laminar separation on the suction surface despite the free-stream turbulent forcing raises the following question: Why does bypass transition not take place upstream 

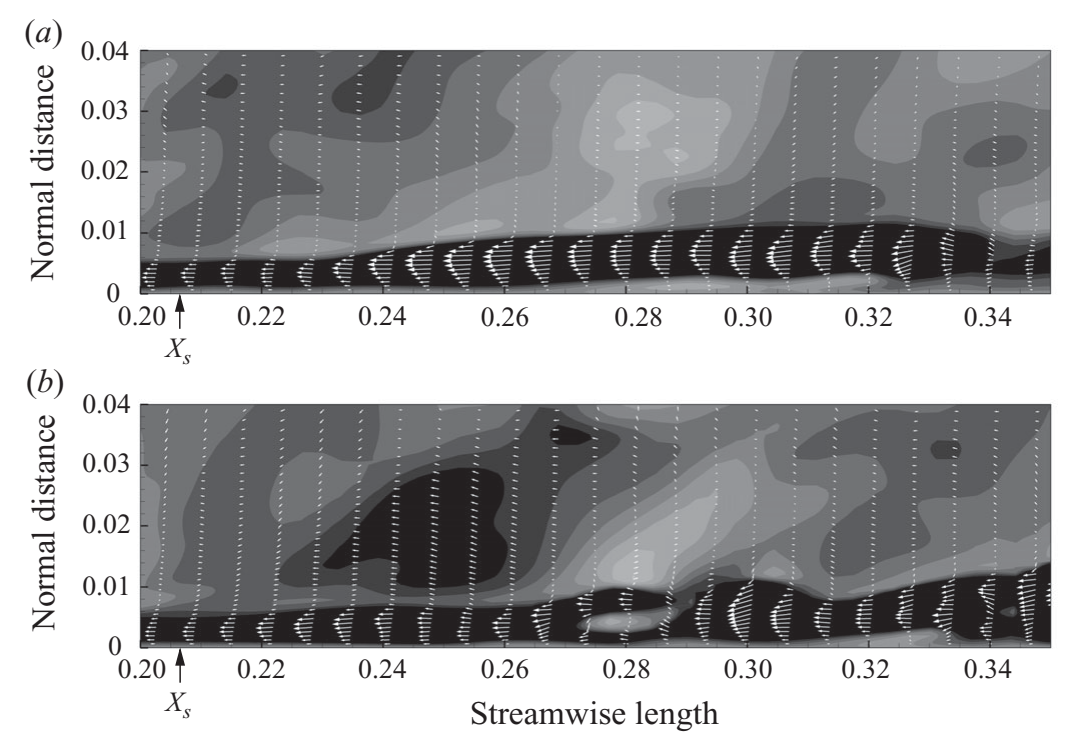

FIGURE 24. Simulation T2. $(a, b)$ Contours of the tangential velocity perturbations, at the first two time instances of figure 23 . The vectors show the in-plane velocity perturbation plotted at every fourth grid point.

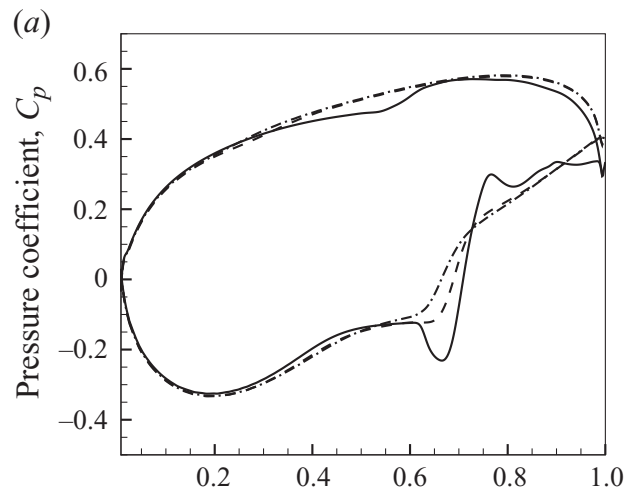

Downstream distance, $x / L$

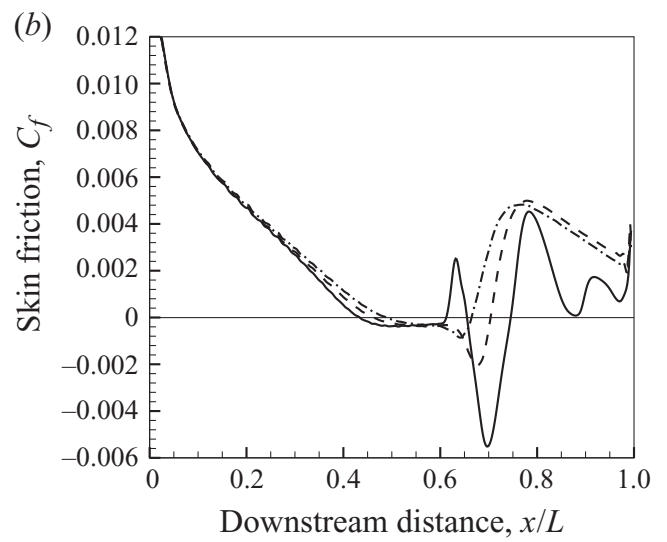

Figure 25. (a) Pressure distribution along the blade surface and $(b)$ skin friction on the suction side for T0 (- $(-)$, T1 (- - ) and T2 (- - - ) .

of separation and, in a similar manner to the pressure surface, promote a reattached boundary layer? It should be recalled that transition takes place on the pressure side at $x / L=0.34$, which is nearly $0.1 L$ upstream of the suction surface separation point. In addition, both blade surfaces share the same free-stream turbulent forcing. An answer is given below, but first the separation behaviour on the suction surface is contrasted for the different levels of free-stream turbulence intensities.

The separation pattern in the absence of free-stream disturbances was discussed in $\S 2$, and was characterized by a main separation region, and a convected, localized separation in the shadow of the shed $\mathrm{K}-\mathrm{H}$ rolls. In that simulation, when $T_{u}=0.0 \%$, the flow remained laminar within the rolls. As the turbulent intensity is increased, the extent of separation is significantly reduced $\left(13.6 \%\right.$ of the axial chord for $\left.T_{u}=6.5 \%\right)$. 
(a)

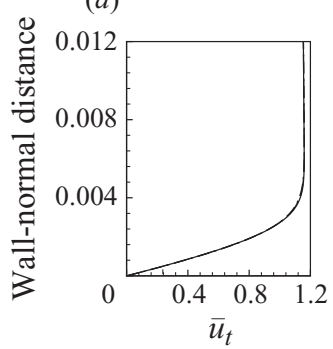

(b)

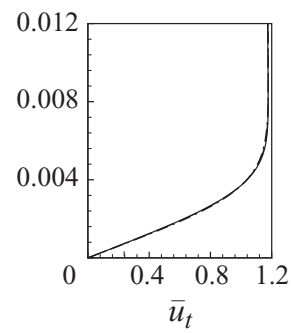

(c)

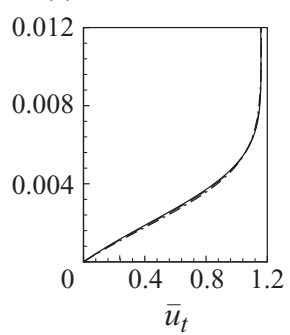

(d)

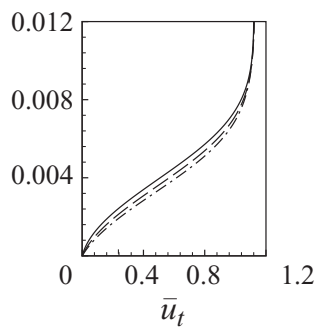

FIgURE 26. $(a-d)$ Profiles of the mean tangential velocity for $0.1 \leqslant x \leqslant 0.4$. Lines: T0; - . $\mathrm{T} 1$ $\mathrm{T} 2$.

(a)

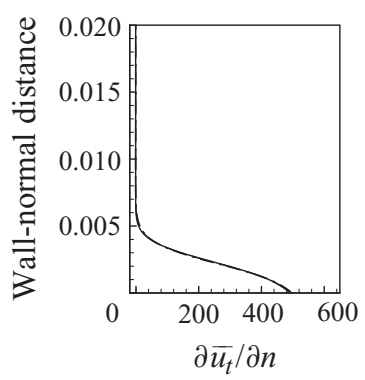

(b)

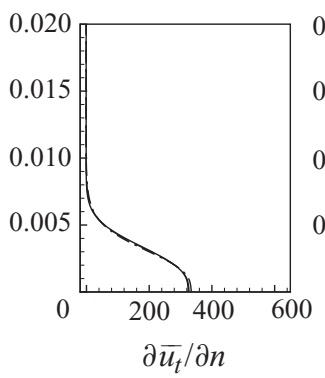

(c)

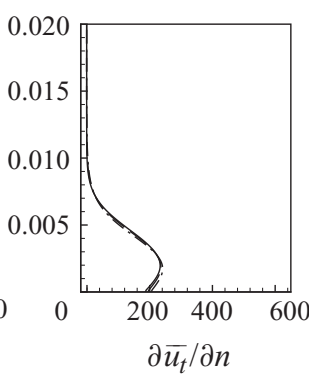

(d)

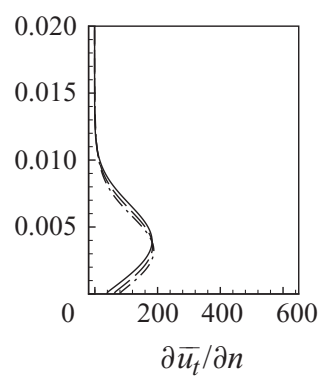

Figure 27. $(a-d)$ The wall-normal gradient of the mean tangential velocity at $0.1 \leqslant x \leqslant 0.4$. Lines: $\_\mathrm{T} 0$; - - $\mathrm{T} 1 ;--\ldots \mathrm{T} 2$.

In addition, separation is delayed. The separation point moves downstream of the laminar location, $x_{s}=0.43$, by $6 \%$ of the axial chord when $T_{u}=6.5 \%$. The departure of the $C_{f}$ curves in figure $25(b)$ from the laminar is small in the favourable pressure gradient region of the suction surface, $x<0.2$, but increases in the adverse pressure region of the blade. The higher free-stream $T_{u}$ conditions promote a slight increase in skin friction, which delays boundary-layer separation. This is further investigated by considering the mean flow and r.m.s. disturbance profiles at various downstream locations.

Figures 26 and 27 contain wall-normal profiles of the mean tangential velocity and mean shear, at downstream locations, $x=\{0.1,0.2,0.3,0.4\}$. Both show a minor variation between the three free-stream conditions. In order to explain the distortion to the mean flow, which delays separation, the quantity $u_{n}^{r m s} \partial \overline{u_{t}} / \partial n$ is plotted at the same downstream stations in figure 28 . This term contributes to the production of the shear stress $\overline{u_{t}^{\prime} u_{n}^{\prime}}$, which represents the turbulent transport of the mean momentum. It is clear that for high free-stream turbulence intensity, the shear-stress production and consequently the momentum transport by wall-normal fluctuations are significantly enhanced inside the boundary-layer shear.

Now the difference in the boundary-layer behaviour on the pressure and suction surface is revisited. The location of bypass transition is known to depend on the amplitude of the Klebanoff distortion, which in turn depends on the intensity of the free-stream forcing and the history of the pressure gradient (Abu-Ghannam \& Shaw 1980). Therefore, one possible cause of the absence of transition on the suction surface is the history of the Klebanoff distortion, and the change in its amplification with 
(a)

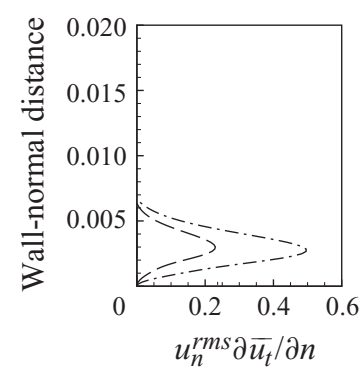

(b)

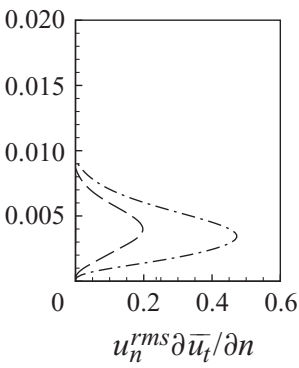

(c)

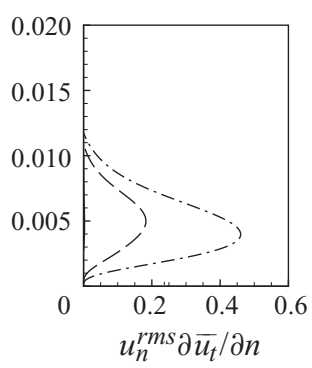

(d)

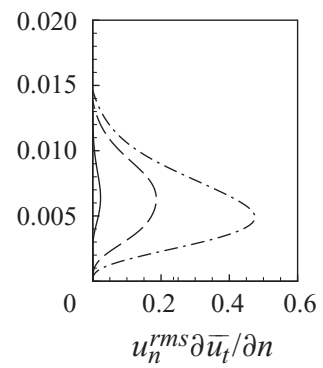

Figure 28. $(a-d)$ Profiles of $u_{n}^{r m s} \partial \overline{u_{t}} / \partial n$ on the suction surface, at $0.1 \leqslant x \leqslant 0.4$. Lines: T0; - - T1; - - - T2.

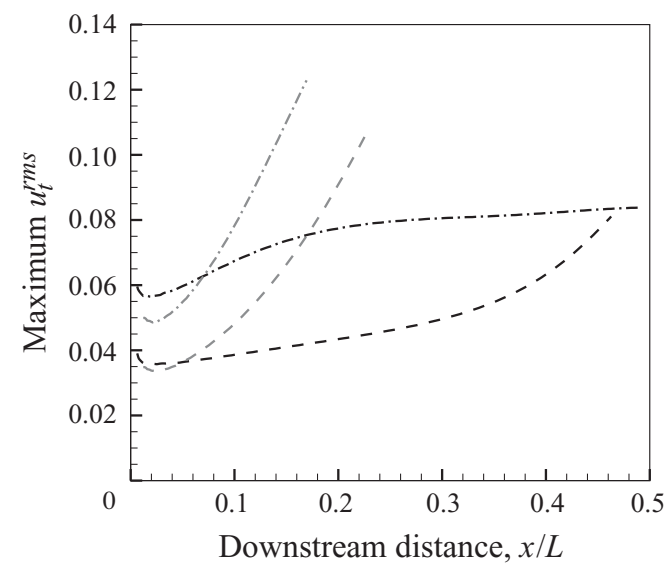

FiguRE 29. Simulations T1 (- - $)$ and T2 (- - - ) : downstream evolution of the maximum $u_{t}^{r m s}$ inside the boundary layer, upstream of separation. Dark lines correspond to the suction surface and light lines correspond to the pressure surface.

pressure gradient (Zaki \& Durbin 2006). Figure 29 shows the maximum $u_{t}^{\text {rms }}$ versus downstream distance, on both the pressure (light lines) and suction surfaces (dark lines), for the two turbulent intensities. Indeed, the Klebanoff distortion amplifies more slowly on the suction surface than on the pressure side.

Profiles of $u_{n}^{r m s} \partial \overline{u_{t}} / \partial n$ on the pressure and suction surfaces are compared in figures 30 and 31 . The results are shown for the $\mathrm{T} 3$ simulation $\left(T_{u}=6.5 \%\right)$, but are representative of the other cases. The peak in the wall-normal shear on the suction surface is larger than on the pressure side of the blade. However, the maximum shear is located at the wall, where $u_{n}^{r m s}$ is identically zero. The term $u_{n}^{r m s} \partial \overline{u_{t}} / \partial n$ is larger on the pressure surface where $u_{n}^{r m s}$ multiplies the maximum shear away from the wall and leads to stronger amplification of Klebanoff streaks. These results are consistent with simulations of bypass transition of flat-plate boundary layers in pressure gradient (Zaki \& Durbin 2006). In the work of Zaki \& Durbin (2006), flow acceleration is observed to reduce the amplification of Klebanoff distortions, and thus to reduce the risk of bypass transition. In the current setting, the same effect is observed along the favourable pressure gradient region of the suction surface, $0<x / L<0.2$. Beyond the point of minimum pressure, Klebanoff distortions do not amplify sufficiently quickly to cause transition to turbulence (see figure 29). Instead, the strong adverse 
(a)

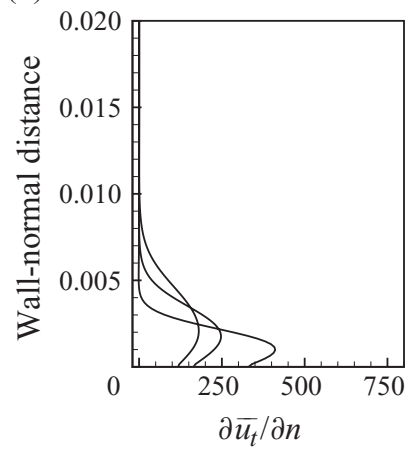

(b)

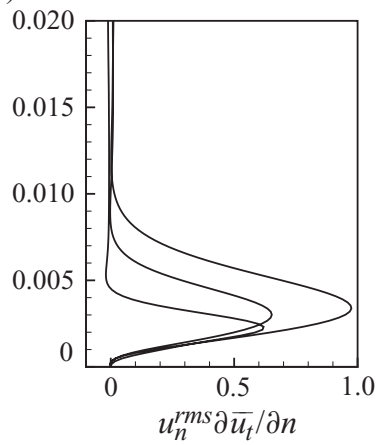

FIgURE 30. Simulation T2, pressure surface. (a) Profiles of $\partial \overline{u_{t}} / \partial n$ and $(b)$ the product $u_{n}^{r m s} \partial \overline{u_{t}} / \partial n$. The three curves correspond to three downstream locations, $x / L=$ $\{0.05,0.10,0.15\}$.

(a)

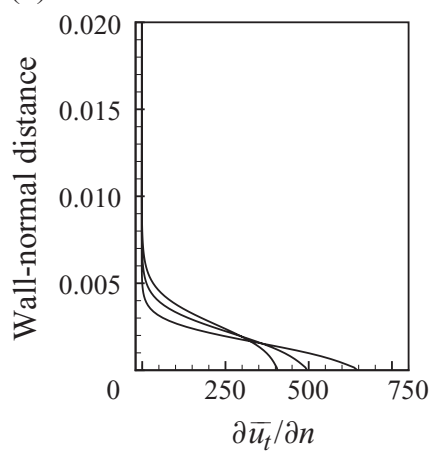

(b)

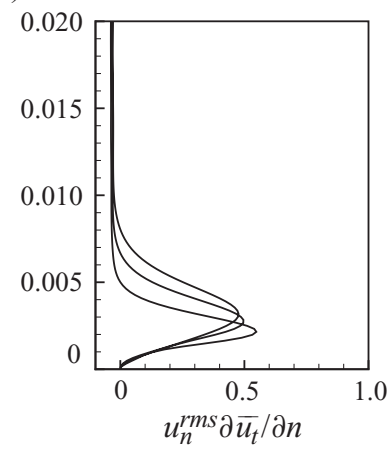

FIgURE 31. Simulation T2, suction surface. (a) Profiles of $\partial \overline{u_{t}} / \partial n$ and $(b)$ the product $u_{n}^{r m s} \partial \overline{u_{t}} /$ $\partial n$. The three curves correspond to three downstream locations, $x / L=\{0.05,0.10,0.15\}$.

pressure gradient causes the mean flow to separate. Had bypass transition taken place, a turbulent boundary layer might have prevented the flow separation.

Based on the time-averaged results of figure 25 , the boundary layer is separated when $T_{u} \leqslant 6.5 \%$. In order to determine whether intermittent attachment takes place, the spanwise-averaged skin friction, $\left\langle C_{f}\right\rangle$, is computed as a function of time. The minimum value of $\left\langle C_{f}\right\rangle$ is plotted in figure 32 ; the flow remains separated for all times, in the spanwise-averaged sense.

The instantaneous flow fields on the suction surface are now inspected for simulations $\mathrm{T} 1$ and $\mathrm{T} 2$ in order to clarify the separation and, where relevant, the transition mechanism at work. The choice of plotting the tangential, wall-normal or spanwise perturbation fields is based on which component best illustrates the phenomenon of interest.

The lowest-intensity case, T1, is considered first. Contours of the tangential velocity perturbations are plotted at a plane $d \sim 1.64 \times 10^{-3}$ away from the wall in figure 33 . The plane is partially opaque in order to allow visualization of surfaces that cross that wall-normal location. The mean separation region is also indicated on the figure. The same time instance is repeated three times: after the first image, the 
(a)

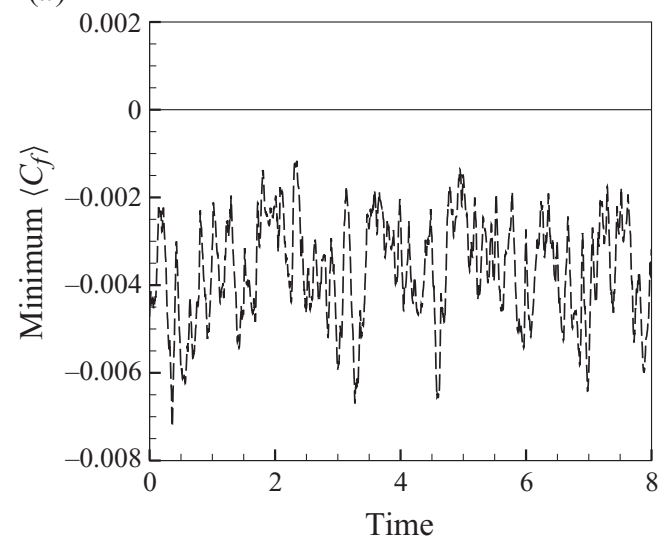

(b)

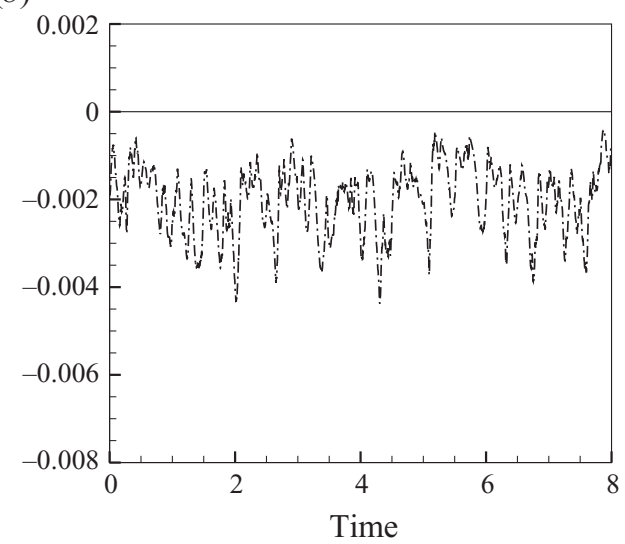

FiguRE 32. The minimum, spanwise-averaged skin friction, $\left\langle C_{f}\right\rangle$ is plotted versus time. The curves correspond to simulations $(a) \mathrm{T} 1$ and $(b) \mathrm{T} 2$. The spanwise-averaged flow for the two lowest free-stream turbulence intensities is separated.

instantaneous separation surface is included, and is visible above and below the plane of $u_{t}^{\prime}$ perturbation contours; then an iso-surface of $\lambda_{2}=-150$ is added.

Previously, the time-averaged results for this case indicated that free-stream turbulence had a negligible effect on the separation onset (see figure 25). Here, the instantaneous perturbation field demonstrates that the Klebanoff streaks inside the boundary layer alter the instantaneous separation surface. The resulting threedimensionality of the separation surface, relative to the mean location, is clear in figure 33(b): separation is shifted upstream where the elongated velocity contours are negative (black), and downstream where they are positive.

The separated shear layer rolls into vortices, but they are not as clearly developed as in the absence of the free-stream turbulence in figure 6. Instead, once the $\mathrm{K}-\mathrm{H}$ roll is formed, it is quickly destabilized by the free-stream turbulence and breaks down. Also, the rolls are not two-dimensional, but are highly modulated in the span. Breakdown to turbulence is followed by reattachment, and the boundary layer remains attached downstream.

Frequency spectra for $\mathrm{T} 1$ are shown in figure 34 . The spectra for the wall-normal perturbation velocity are shown in figure 34(a) and for the tangential component are shown in figure $34(b)$. The laminar shedding frequency, $F=175$, is shown in the figure by a dashed line. Rather high energy content is observed near $F \sim 200 \rightarrow 240$, in the spectra of the wall-normal disturbance. However, this frequency range is not distinguished in the streamwise disturbance spectra. Instead, they capture the generation of the low-frequency Klebanoff disturbance, which amplifies in the adversepressure-gradient region $x / L>0.2$.

According to the averaged results, $\mathrm{T} 2$ also exhibits laminar separation and subsequent turbulent reattachment. The time evolution of the minimum $\left\langle C_{f}\right\rangle$ also predicted a fully separated flow. Here, the instantaneous velocity fields are examined in order to highlight possible flow instabilities and their interaction with the separated boundary layer.

A time series of the instability is shown in figures 35 and 36 . The first sequence, figure 35, shows the wall-normal perturbation field near the suction surface. In figure 35(a), a wavepacket is seen to develop upstream of the mean separation surface. 

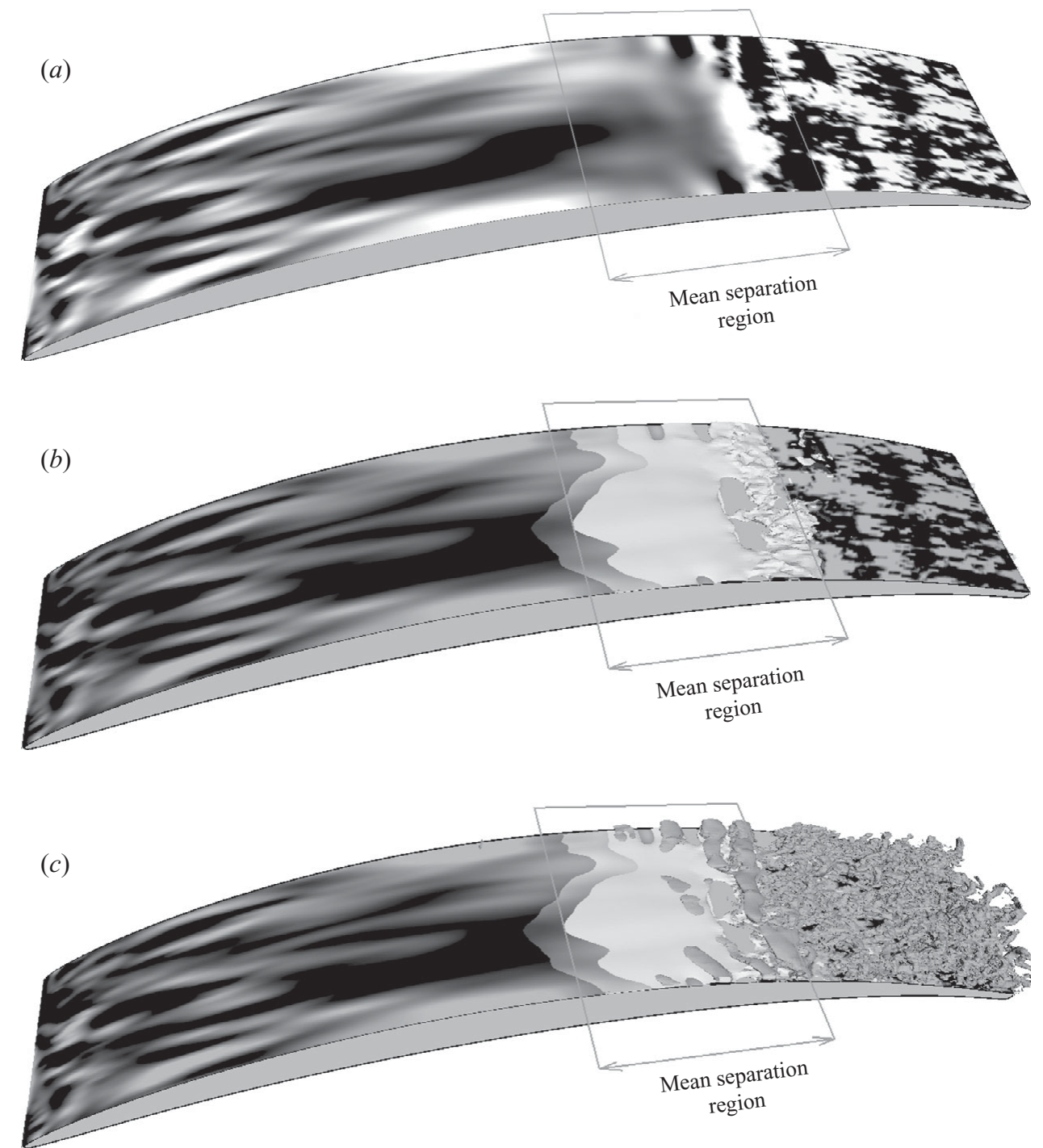

FIGURE 33. Simulation T1: separation on the suction surface of the blade. The same time instance is repeated three times. In $(a)$, light and dark contours correspond to positive and negative tangential disturbances $-0.05<u_{t}^{\prime}<0.05$. In $(b)$, the three-dimensional separation surface (white region) is superimposed on the perturbation field. The separation surface is modulated by the boundary-layer perturbations. In $(c)$, the iso-surface $\lambda_{2}=-150$ is also included.

As it convects downstream, it breaks down to turbulence. The spreading turbulent spot, as time progresses, occupies a larger region of the blade surface. Figure 35(c, $d)$ shows the spot merging with the downstream, fully turbulent boundary layer. The same figure also shows the formation of another instability. This wave emerges, however, within the mean separation region. It retains a quasi two-dimensional form until a late stage, prior to merging with the downstream turbulent flow.

The same time series is repeated in figure 36, for the streamwise velocity disturbance. It is evident from the figure that the streaks in T2 are much narrower in the spanwise direction than those in T1. Therefore, the modulation of the separation surface in the 
(a)

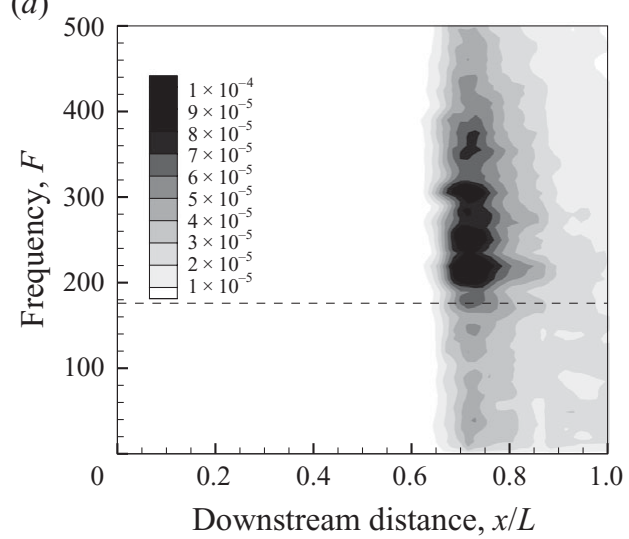

(b)

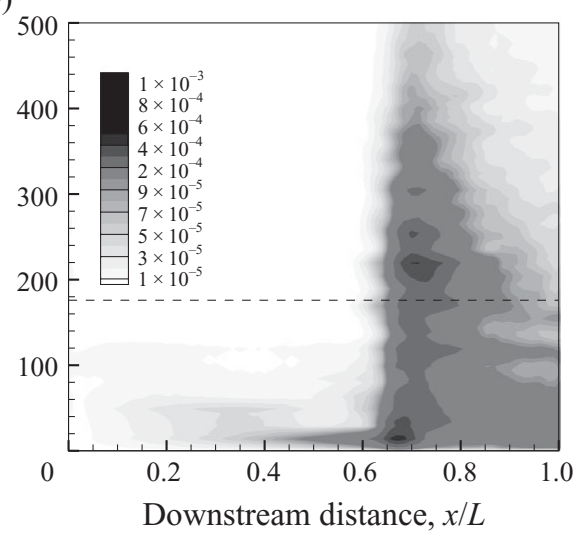

FIGURE 34. Simulation T1: frequency spectra at the wall-normal location where the disturbance energy is the maximum. The contours are of the power spectral density computed from $(a)$ the wall-normal and $(b)$ streamwise perturbation velocities.

spanwise direction is at a much finer scale. The figure also shows the instantaneous separation surface. It is now apparent that the averaged results presented earlier, which suggest that the boundary layer is fully separated, are misleading. The boundary layer on the suction surface is attached in regions where turbulent spots are formed. It is also interesting to note that the instability wave which emerges upstream of the mean separation location (figure 36a) initially triggers a mild local separation. At this stage, the instability wave has the form of a small spanwise roll, whose strength is sufficient to separate the local velocity profile. However, as the instability moves downstream, it breaks down to turbulence and causes a growing region of attached flow. The attachment region increases in the spanwise extent as the spot spreads. In contrast, the second instability, which emerges in figure 36(c,d), does not result in significant attachment, due to its late inception.

A time series of the skin friction at the location marked ' $\mathrm{x}$ ' in figure $35(a)$ was recorded and is shown in figure 37. The times corresponding to figure $35(a, d)$ are denoted by $A$ and $B$, respectively. Since the recording station lies within the mean separation region, $C_{f}$ first has a negative value. As the spot convects past the sensing station, a strong fluctuation is observed, and is associated with the boundary layer being buffeted by the patch of turbulence. Beyond the final snapshot of figure 35, the convected spot has cleared the recording location. The skin friction in figure 37, beyond point $B$, slowly relaxes to the separated state. This relaxation is known as the calming effect that trails turbulent spots. One explanation of calming is a relaxation of the boundary layer from a turbulent condition, to a laminar state. However, if one considers the time interval $t \in[A, B]$, the averaged $C_{f}^{A B} \sim 0.0004$, which is significantly smaller than the turbulent level for this flow, which is $O(0.004)$. Therefore, the calmed region observed in our simulations is not a relaxation of the boundary layer, but rather a region that trails the turbulent spots and that is more stable than the undisturbed flow.

\subsection{The influence of high free-stream turbulence intensity (T3, T4)}

The state of the boundary layer on the suction surface for T3 and T4 is also discussed starting with the averaged quantities, followed by instantaneous perturbation fields. The pressure and skin-friction coefficients for these cases are included in figure 38, along with the curves for $\mathrm{T} 1$ and $\mathrm{T} 2$. As the turbulence intensity is increased to 

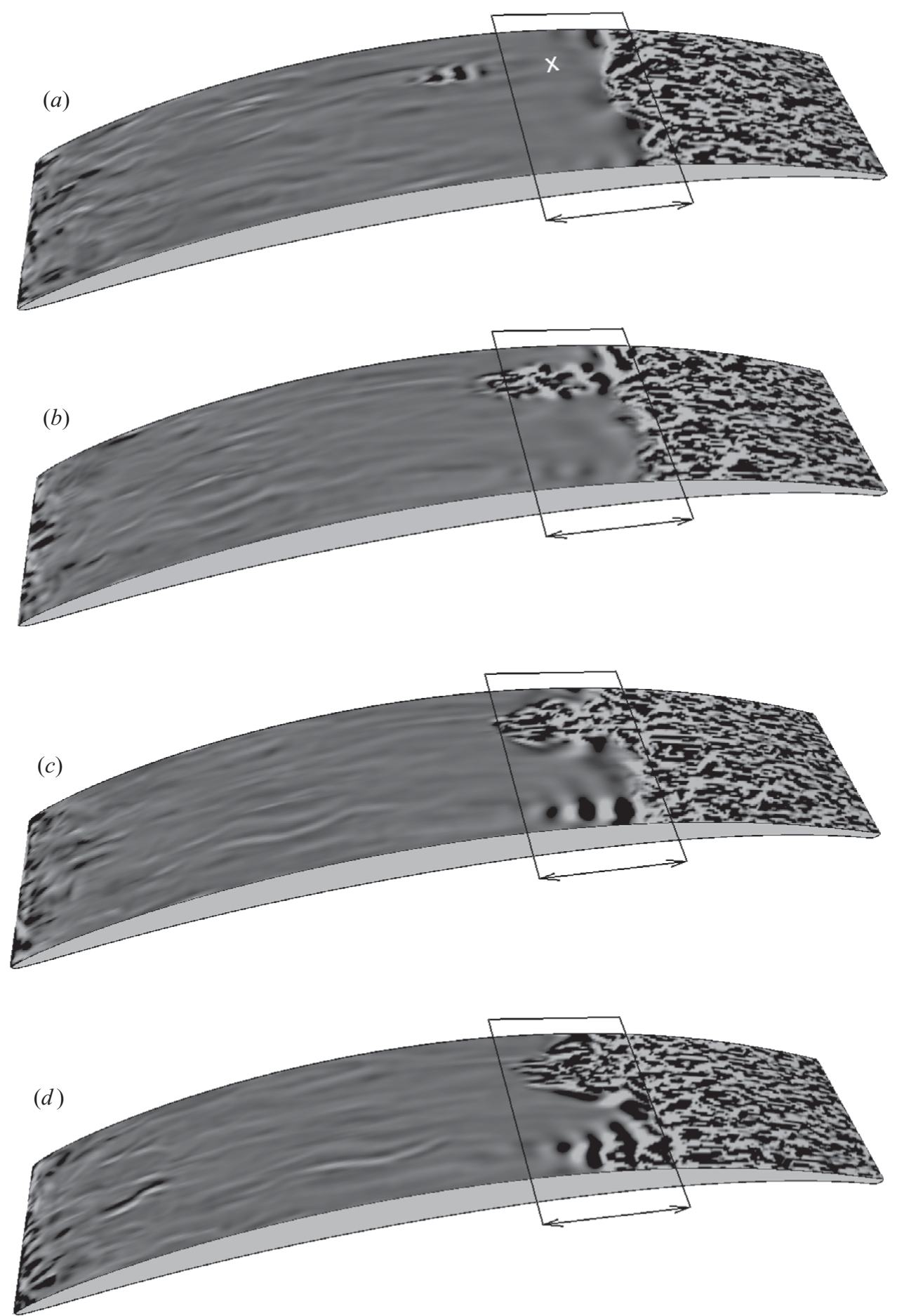

FIgURE 35. Simulation T2. $(a-d)$ Contours of the normal velocity perturbations, $-0.01<u_{n}^{\prime}<0.01$, inside the suction surface boundary layer, at a distance $d \sim 1.64 \times 10^{-3}$ from the wall. The mean separation length is shown by dark lines. The sequence spans a time period $\Delta t=0.4$. 

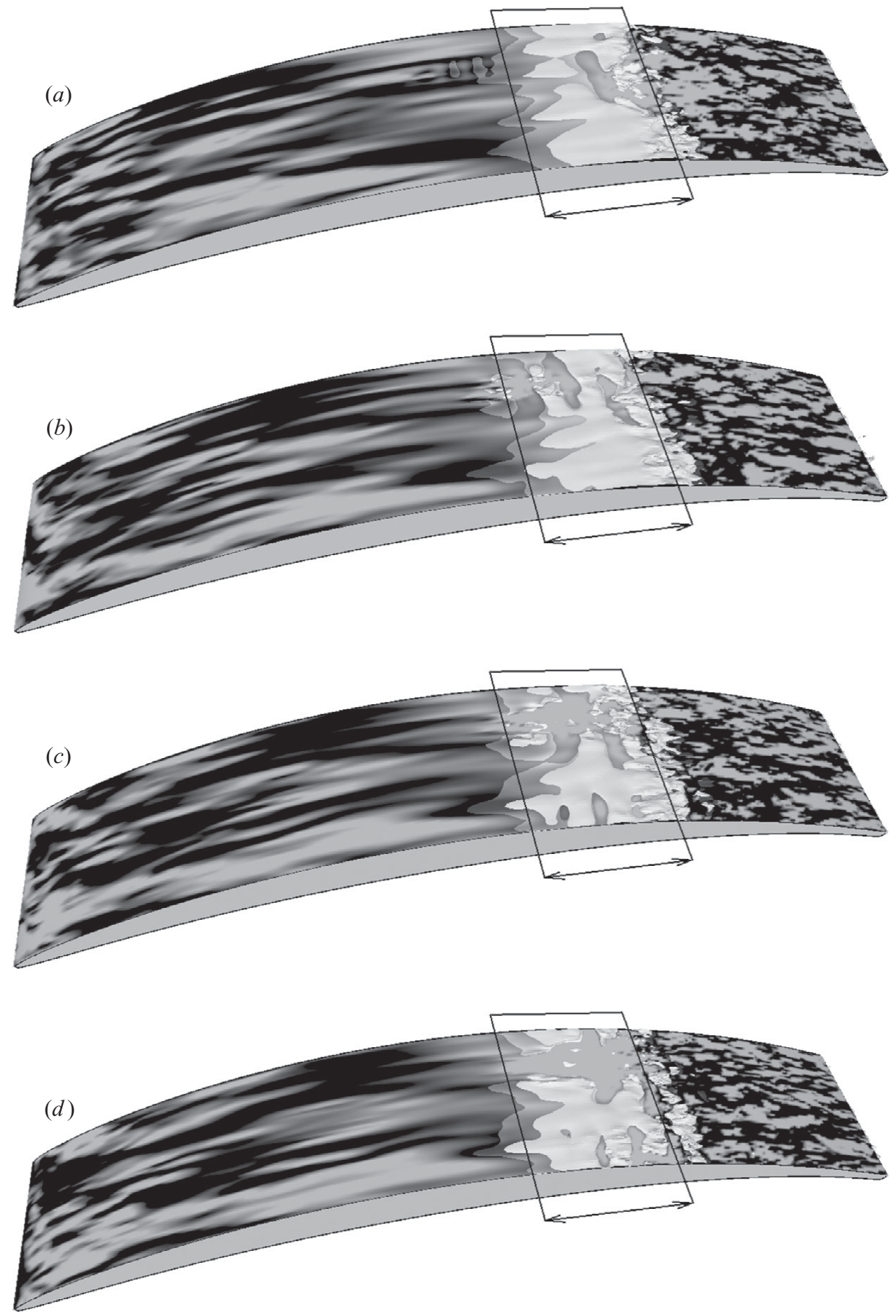

Figure 36. Simulation T2. $(a-d)$ Contours of the tangential velocity perturbations, $-0.05<u_{t}^{\prime}<0.05$, with the instantaneous separation surface superimposed. The mean separation length is shown by dark lines. The sequence is at the same times as in figure 35 . 


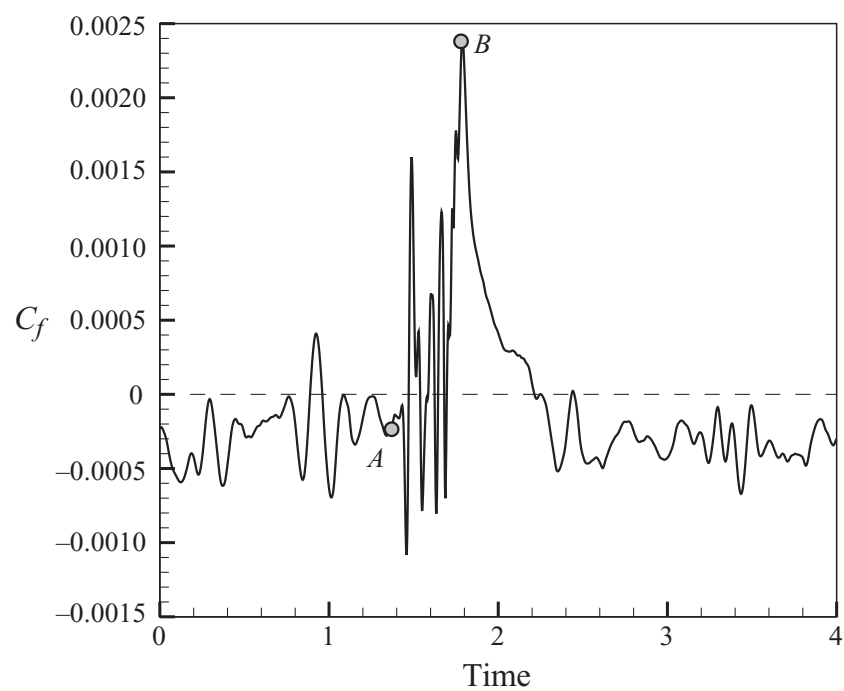

FIGURE 37. Simulation T2: the time-dependent skin friction, $C_{f}(t)$, due to the streamwise velocity component, evaluated at $(x, z)=(0.55,0.055)$. This location on the blade surface is identified by ' $\mathrm{x}$ ' in figure $35(a)$. The time trace shows the calming effect associated with the passing of the turbulent spot in figures 35 and 36.

(a)

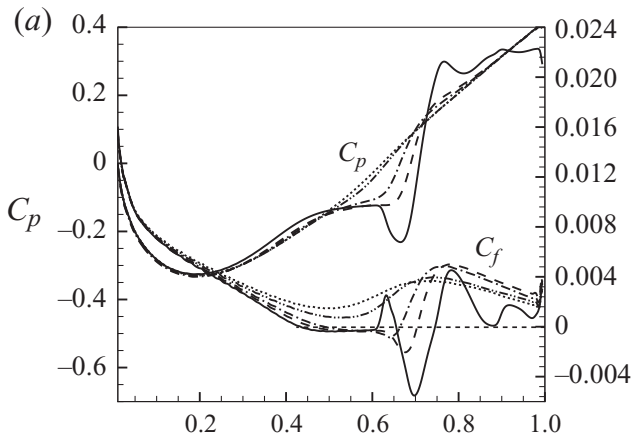

Downstream distance, $x / L$

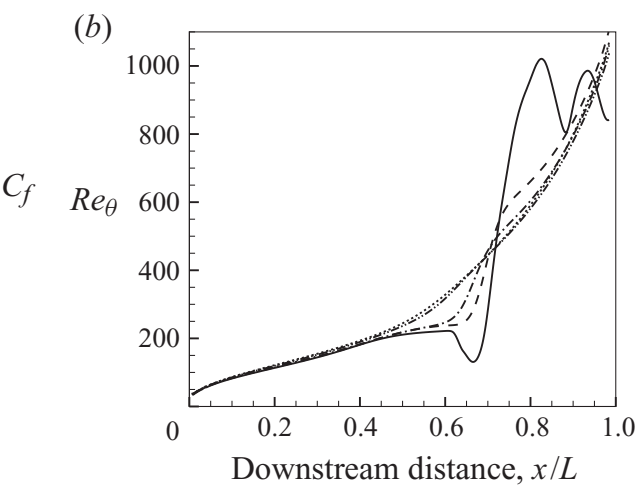

FIgURE 38. (a) The pressure coefficient, $C_{p}$, and the skin friction, $C_{f}$, along the suction surface. (b) The downstream variation of the momentum thickness Reynolds number, $R_{\theta}$. Lines: -

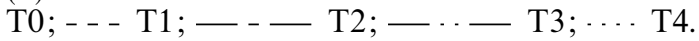

$T_{u}=8.0 \%$ (case T3), the suction side boundary layer no longer separates. The skin friction curve shows transition to turbulence at this, and at higher, $T_{u}$ levels. The minimum in the skin friction, however, is achieved beyond the laminar separation location. This is possible because of a combination of separation delay, due to the distortion of the base flow discussed above, and higher Klebanoff mode amplitude which promotes transition at increasing free-stream turbulence levels. The downstream evolution of the Reynolds number, based on the momentum thickness, is also reported in figure 38. The onset of transition at the two highest $T_{u}$ levels is at $R e_{\theta}=255$ and the slightly upstream location $R e_{\theta}=245$, respectively. The lack of sensitivity of the transition location at high free-stream turbulence levels is consistent with the experimental observations reported by Abu-Ghannam \& Shaw (1980). However, transition in our simulations is downstream of the $R e_{\theta}$ predicted by their data correlation. The same trend is observed in the experimental data by Abu-Ghannam \& 


$\begin{array}{cccccc}T_{u}(\%) & 0.00 & 3.25 & 6.50 & 8.00 & 10.00 \\ \mathscr{A}(\%) & 0.0 & 0.0 & 0.0 & 59.8 & 96.6\end{array}$

TABLE 2. The percentage of time $\mathscr{A}$ where the spanwise-averaged flow on the suction surface is attached.
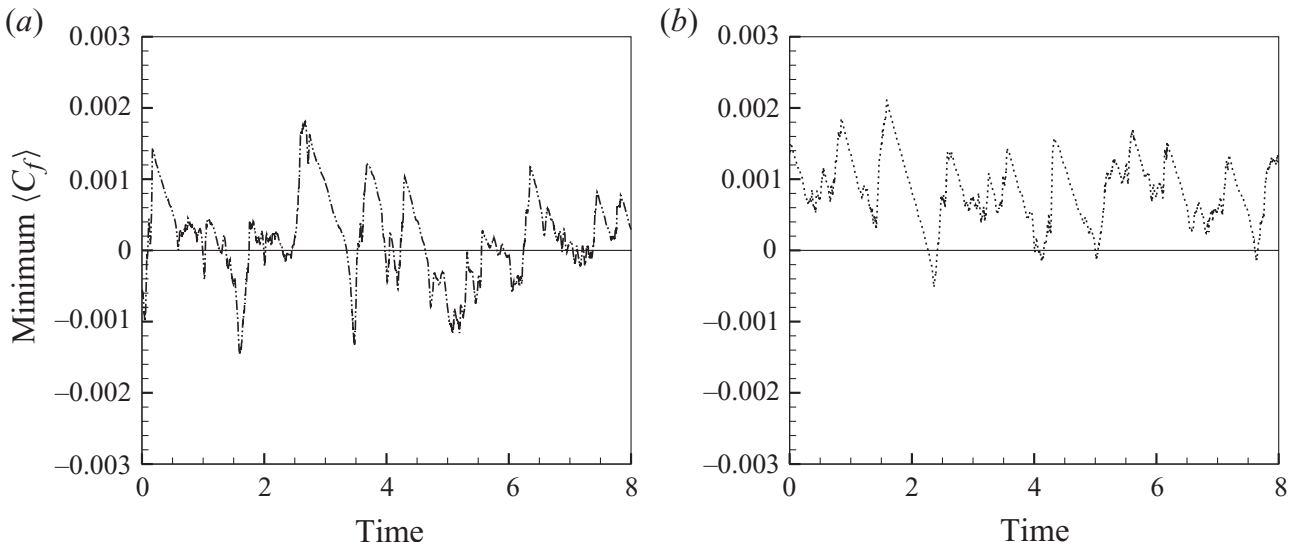

FiguRE 39. The minimum, spanwise-averaged skin friction, $\left\langle C_{f}\right\rangle$, is plotted versus time. The curves correspond to $(a)$ T3 and $(b)$ T4. For simulation T3, the flow is intermittently attached, and mostly attached for the highest free-stream turbulence intensity, T4.

Shaw (1980), which have a larger transition $R e_{\theta}$ than their correlation at $T_{u}=4.8 \%$. They attributed the deviation to the pressure gradient history. In the flow studied herein, the variation in the pressure gradient is most pronounced along the suction surface of the blade.

The minimum in the spanwise-averaged skin friction is plotted versus time in figure 39, for T3 and T4. The percentage of time that the flow is attached, $\mathscr{A}$, is also reported in table 2 . The flow is only attached $59 \%$ of the time for T3 - separation is therefore not entirely prevented, but is rather intermittent. This observation motivates investigation of the instantaneous velocity field on the suction surface of the blade.

A snapshot of the suction surface from T3 is provided in figure 40. The contours show the wall-normal velocity perturbation. The single snapshot demonstrates the presence of small regions of separation near the laminar-turbulent boundary. However, the boundary layer is predominantly attached. Also marked in the figure is an instability wave, similar to that previously recorded in T2, but which takes place early upstream at the higher $T_{u}$ level. This instability becomes turbulent later in time. The generation of disturbance waves, similar to the marked structure in figure 40 , does not necessarily lead to breakdown. During the simulations, events of such appearance initially amplify, but can subsequently decay without becoming turbulent. These waves, travelling at relatively lower phase speeds, are overtaken by the faster travelling Klebanoff modes. The influence of Klebanoff distortions on these waves has been addressed in the literature, with conflicting conclusions. For example, Cossu \& Brandt (2002) suggest that the distortions can stabilize instability waves. Others have shown that unsteady, jetlike distortions enhance the secondary instability of the waves (Liu et al. 2008b). Wu \& Choudhari (2003) have derived an enhancement to the growth rate of the instability. These competing mechanisms, therefore, determine the fate of these disturbances, be it breakdown to turbulence or decay. 


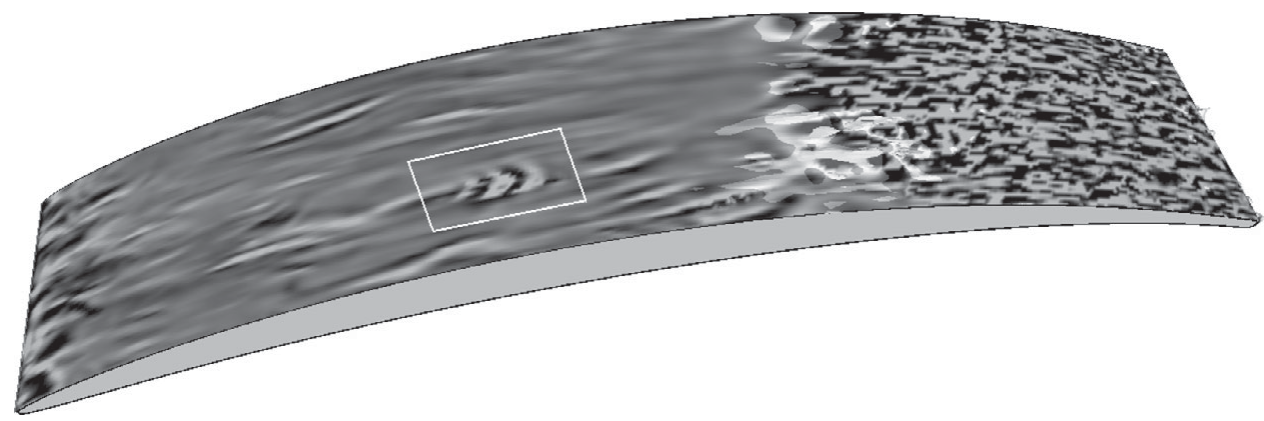

FIGURE 40. Simulation T3: contours of the wall-normal velocity perturbations $-0.01<u_{n}^{\prime}<0.01$ at a distance $d \sim 1.64 \times 10^{-3}$ from the suction surface. The highlighted perturbation amplifies and leads to breakdown, in a manner similar to the instability in figure 35 for simulation T2.

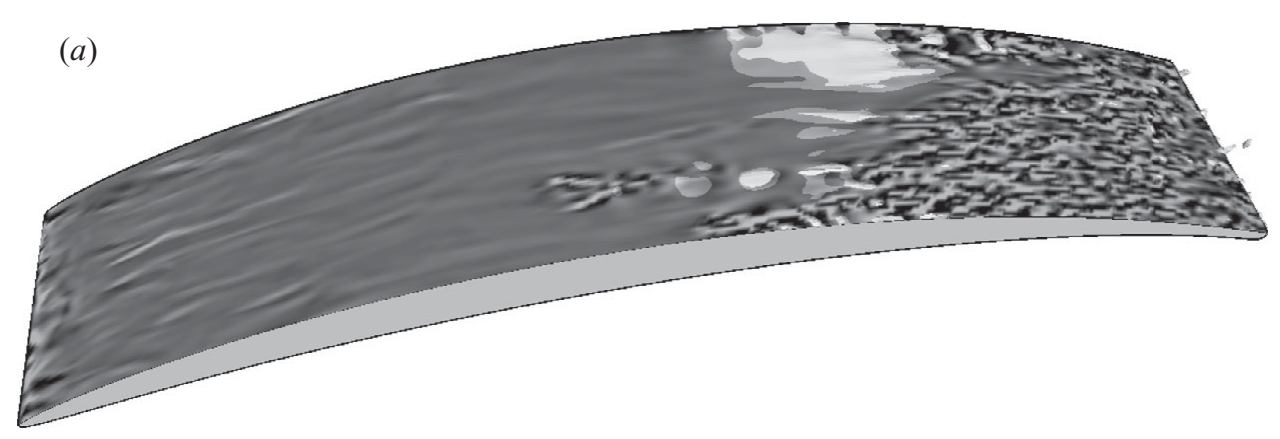

(b)

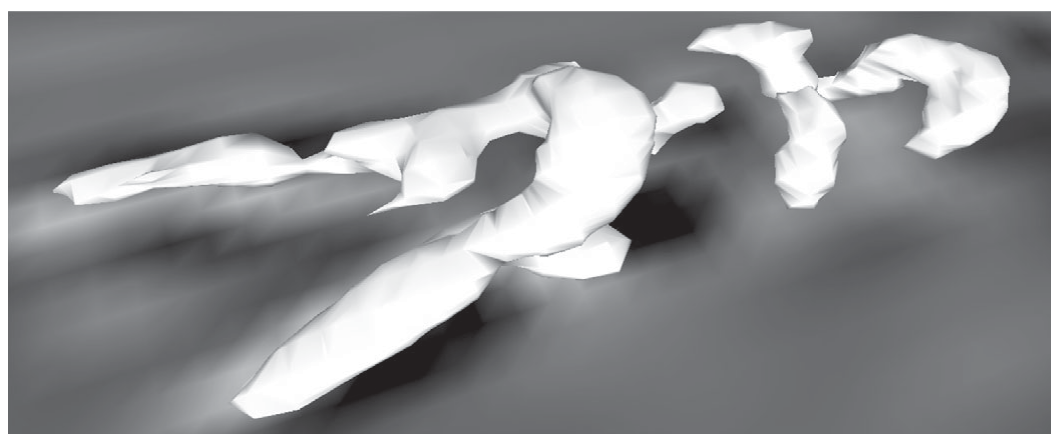

FIGURE 41. Simulation T3. (a) Contours of the normal velocity perturbations $-0.01<u_{n}^{\prime}<0.01$. (b) A zoomed-in view of the $\Lambda$-structure which precedes breakdown. The $\Lambda$-structure is identified by an iso-surface, $\lambda_{2}=-390$.

Similar to the pressure surface of the blade, it is expected that adverse pressure gradient on the suction surface promotes the amplification of discrete instability waves and naturally triggered instabilities. A formation of $\Lambda \mathrm{s}$ is often an indication of the presence of such instabilities. One example from simulation T3 is shown in figure 41. Figure 41(a) shows the perturbation field near the blade's surface. 

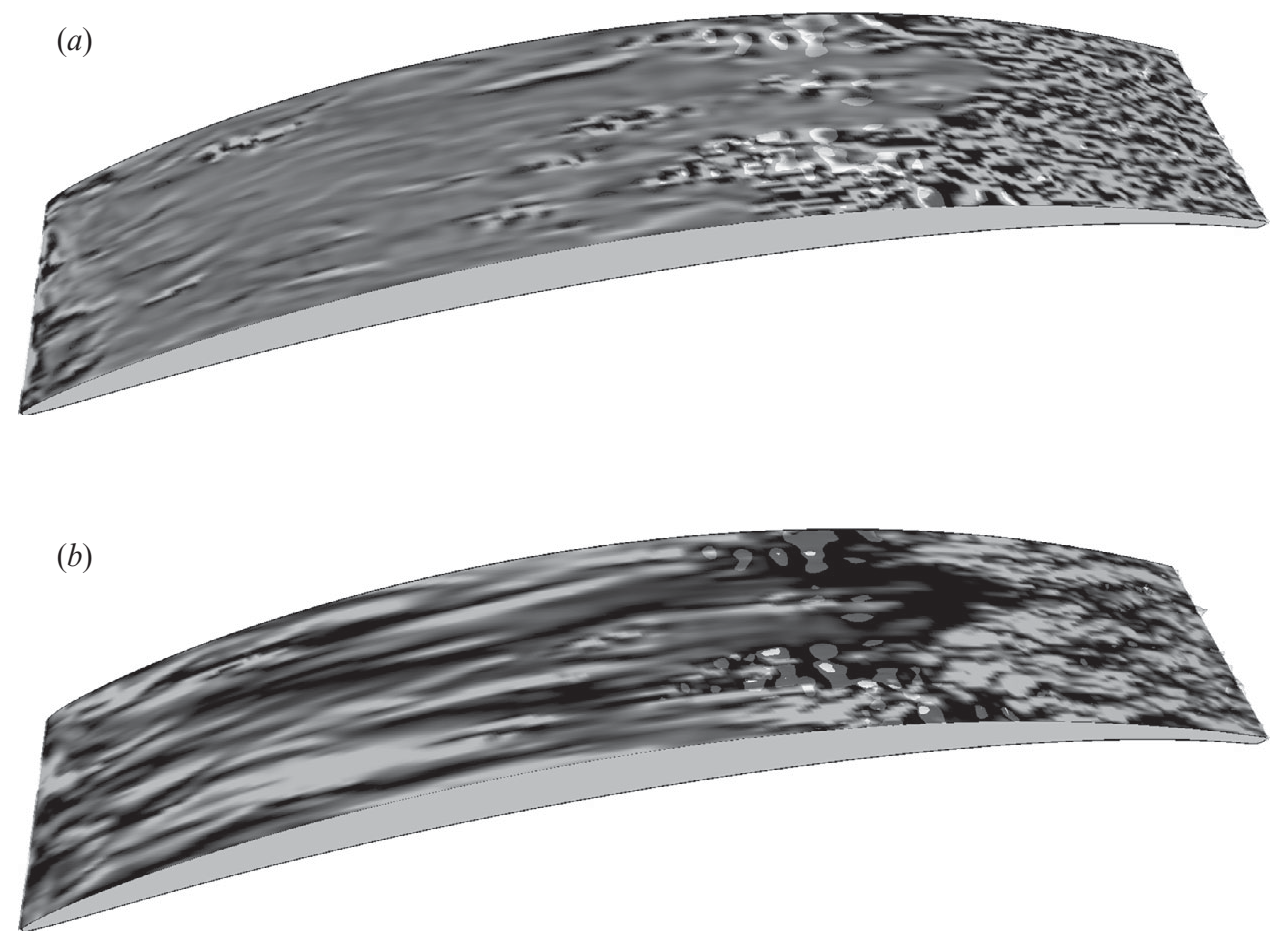

FigURE 42. Simulation T4: contours of $(a)$ the normal $-0.01<u_{n}^{\prime}<0.01$ and $(b)$ tangential $-0.1<u_{t}^{\prime}<0.1$ velocity perturbations. A large number of turbulent spots are observed to emerge due to the boundary-layer forcing by the higher free-stream turbulence intensity.

Figure $41(b)$ is a zoomed-in view, with the iso-surface $\lambda_{2}=-390$ superimposed. The figure distinctly confirms that natural transition mechanisms, analogous to those studied in canonical flows (Herbert 1988; Liu et al. 2008a), are indeed relevant in complex flow conditions.

The spanwise-averaged skin friction predicts that the suction surface boundary layer in case T4 is attached $96.6 \%$ of the time (figure 39). This is primarily due to a high frequency of the spot inception, in response to the high level of free-stream turbulence. A snapshot of the boundary layer, demonstrating the higher spot formation rate in $\mathrm{T} 4$, is shown in figure 42. The mechanisms underlying the formation of these spots are of the bypass type. It is remarkable that such a high turbulence intensity is required in order for the bypass mechanism to dominate. The current simulations demonstrate that classical, discrete instability waves can continue to play a role even at high background turbulence levels.

The spectra in figure 43 confirm that case $\mathrm{T} 4$ is dominated by pure bypass transition. These spectra are dominated by a range of low-frequency disturbances. Thus, the boundary layer is perturbed by a spectrum of Klebanoff modes. This should be contrasted to T1 (figure 34) where only a very narrow range of low-frequency disturbances has sufficient energy to be captured by the contour levels. Also, in case $\mathrm{T} 4$, the transition region is not as distinctly demarcated, due to the buffeted nature of the boundary layer. 


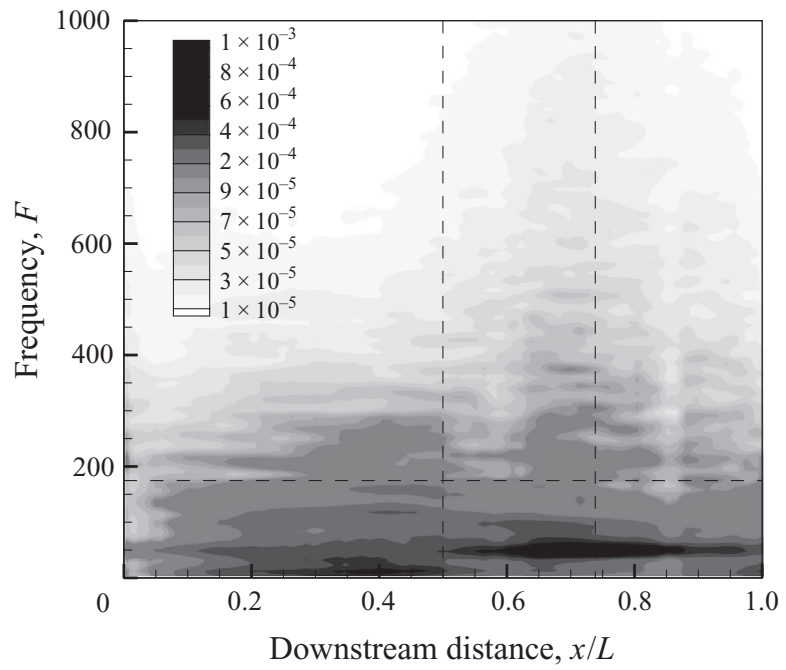

FIGURE 43. Simulation T4: frequency spectra at the wall-normal location where the disturbance energy is the maximum. The power spectral density is computed from the streamwise velocity perturbation. The vertical dashed lines mark the onset and end locations of transition to turbulence. The horizontal line marks the shedding frequency of the laminar separation bubble from $\mathrm{T} 0$.

\section{Conclusion}

Direct numerical simulations of transitional flow on the NACA 65 aerofoil in a linear compressor passage were carried out with various levels of free-stream turbulence intensities. The numerical experiments are rich with fundamental transition phenomena, due to the vortical forcing and the adverse pressure gradient.

In the absence of free-stream forcing, the pressure surface boundary layer undergoes laminar separation. When vortical perturbations are introduced at the inlet of the computational domain, they cause the boundary layer to transition to turbulence. The transition location, being upstream of the laminar separation point, ensures that the flow remains attached. Despite the concave curvature of the pressure surface, transition is not via Görtler vortices. Instead, at the inlet turbulence intensity $T_{u}=3.25 \%$, streamwise elongated disturbances were amplified upstream of transition. Their breakdown, however, did not follow the conventional bypass mechanism, where lifted low-speed shear layers are perturbed by the free-stream, high-frequency forcing. Instead, an inner instability was observed, and its later stages has the form of $\Lambda$ structures, which resemble those found in the secondary instability of classical T-S waves.

The velocity profile upstream of transition was extracted and a local, linear stability analysis was performed in order to answer whether the wavenumber of $\Lambda \mathrm{s}$ can be related to instability waves of the base flow. The velocity profile indeed possesses a discrete instability at $\alpha=125$, which corresponds to the inner mode. The current study, therefore, highlights the significance of earlier fundamental studies of the interaction of Klebanoff distortions and T-S waves (Cossu \& Brandt 2002; Fasel 2002; Fransson et al. 2005; Liu et al. 2008a). At higher free-stream turbulence levels, bypass transition via the secondary instability of streaks becomes dominant. The transition mechanism therefore agrees with previous numerical and physical 
experiments of bypass transition, in the absence of pressure gradient and on turbine blades (for example Jacobs \& Durbin 2000; Hernon et al. 2007).

The co-existence of Klebanoff modes and instability waves also highlights the potential importance of the spectral make-up of the incoming turbulence, and its influence on transition. Traditionally, the high- and low-frequency components of the free-stream turbulence have been addressed separately, in connection with natural and bypass transition, respectively. The relatively higher-frequency disturbances were the focus of receptivity studies, in relation to the generation of $\mathrm{T}-\mathrm{S}$ waves (Saric, Reed \& Kerschen 2002); the lower-frequency content was investigated in the context of the generation of Klebanoff modes (Leib, Wundrow \& Goldstein 1999). It was also suggested that the bypass mechanism dominates at turbulence intensities greater than $1 \%$. In the current study of a compressor blade, both the bypass and instability-wave mechanisms are observed simultaneously, but a shift in the dominant mechanism is possible for different spectral make-up of the incoming turbulence.

The suction side boundary layer also undergoes laminar separation in the absence of free-stream vortical disturbances. Kelvin-Helmholtz rolls are shed from the separated shear layer and remain non-turbulent as they convect downstream. They maintain a region of separated flow in their shadow, on the surface of the blade, thus preventing full reattachment of the boundary layer.

When vortical disturbances are introduced at the inlet, separation is not immediately bypassed. Instead, the flow behaviour is dependent on the free-stream turbulence intensity. At the lowest intensity, $T_{u}=3.25 \%$, separation persists, and is modulated by the boundary-layer streaks. The formation of $\mathrm{K}-\mathrm{H}$ rolls is immediately followed by breakdown to turbulence due to the perturbations in the free stream. As the perturbation level is increased, $T_{u}=6.5 \%$, turbulence spots are observed on the suction surface. Their interaction with the separated boundary layer resulted in local reattachment and the calming effect was observed. Finally, at the highest-intensity cases, $T_{u}>6.5 \%$, a combination of mean flow distortion and transition to turbulence caused the boundary layer to remain attached.

After converged statistics were computed from simulations T1-T4, the inlet perturbations were ceased. The computations were sustained with only the mean flow prescribed at the inlet plane, in order to verify whether the flow recovers the state found in simulation T0. On the pressure surface, separation was re-established and resembled the flow state in T0. On the suction surface, however, the flow did not recover the separation characteristics of T0. Instead, the laminar separation continued to undergo turbulent reattachment, despite the absence of any fluctuations in the free stream.

The origin of an absolute instability on the suction surface was examined, and is similar to the mechanism reported by Jones et al. (2008). It is best illustrated using the instantaneous fields from simulation T0. Figure 44 is a zoomed-in view of the last phase of the laminar shedding cycle originally shown in figure 6 . The contours of spanwise vorticity clearly show the $\mathrm{K}-\mathrm{H}$ rolls. Superimposed on the vorticity are iso-contours of streamwise velocity. The reverse flow from the downstream roll can convect perturbations upstream into the braid region. These perturbations are subsequently convected farther upstream by the subsequent roll. Therefore, when the inlet fluctuations were removed from simulations T1-T4, this mechanism sustained breakdown of the shed vortices and caused turbulent reattachment of the separated boundary layer.

This paper has focused on the interaction of free-stream turbulence with the boundary layers in a compressor passage. The role of base-flow unsteadiness was 


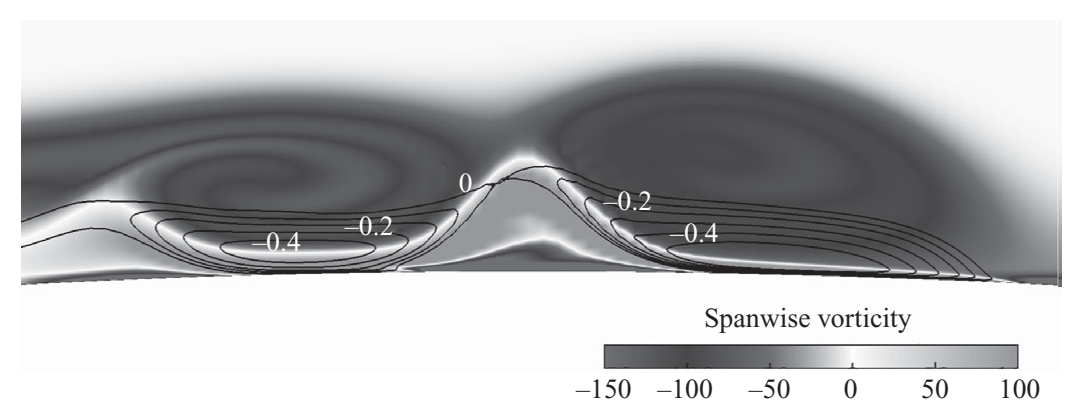

FIGURE 44. Simulation T0: contours of the spanwise vorticity on the suction surface. The snapshot corresponds to the marked region in figure $6(d)$. The lines mark contours of the tangential velocity.

not discussed. In a separate effort, we have investigated the effect of migrating turbulent wakes (Zaki et al. 2009). In that case, the free stream was laminar between consecutive wakes, and therefore the turbulent forcing was intermittent. Only one wake-turbulence intensity was simulated, $T_{u}=3.5 \%$. The suction surface boundary layer underwent laminar separation and turbulent reattachment, similar to the current case T1. In between wakes, the separation location moved upstream and the extent of the separation region increased. Further simulations are currently being performed in order to determine the influence of mean-flow unsteadiness and higher turbulence levels within the migrating wakes.

The authors would like to thank the steering committee of the Super Computing Facilities in Bavaria (HLRB) for granting computing time on the Hitachi SR8000-F1 at the Leibniz Computing Centre (LRZ) in Munich. T.Z. would like to acknowledge support from the Air Force Office of Scientific Research (FA8655-07-1-3009).

\section{REFERENCES}

Abdessemed, N., Sherwin, S. J. \& Theofilis, V. 2009 Linear instability analysis of low-pressure turbine flows. J. Fluid Mech. 628, 57-83.

Abu-Ghannam, B. J. \& Shaw, R. 1980 Natural transition of boundary layers: the effects of turbulence, pressure gradient and flow history. J. Mech. Engng Sci. 22, 213-228.

Alam, M. \& SANDham, N. D. 2000 Direct numerical simulation of short laminar separation bubbles with turbulent reattachment. J. Fluid Mech. 410, 1-28.

Andersson, P., Berggren, M. \& Henningson, D. S. 1999 Optimal disturbances and bypass transition in boundary layers. Phys. Fluids 11 (1), 134-150.

Brandt, L., Schlatter, P. \& Henningson, D. S. 2004 Transition in boundary layers subject to free-stream turbulence. J. Fluid Mech. 517, 167-198.

Butler, K. M. \& FARrell, B. F. 1992 Three-dimensional optimal perturbations in viscous flow. Phys. Fluids 4 (8), 1637-1650.

Corke, T. C. \& Gruber, S. 1996 Resonant growth of three-dimensional modes in Falkner-Skan boundary layers with adverse pressure gradients. J. Fluid Mech. 320, 211-233.

Cossu, C. \& BrandT, L. 2002 Stabilization of Tollmien-Schlichting waves by finite amplitude optimal streaks in the Blasius boundary layer. Phys. Fluids 14 (8), L57-L60.

FASEL, H. F. 2002 Numerical investigation of the interaction of the Klebanoff-mode with a TollmienSchlichting wave. J. Fluid Mech. 450, 1-33.

Fransson, J., Brandt, L., Talamelli, A. \& Cossu, C. 2005 Experimental study of the stabilization of Tollmien-Schlichting waves by finite amplitude streaks. Phys. Fluids 5, 1-15.

Goldstein, M. E. \& LeE, S. S. 1992 Fully coupled resonant-triad interaction in an adverse-pressuregradient boundary layer. J. Fluid Mech. 245, 523-551. 
Goldstein, M. E. \& Sescu, S. 2008 Boundary-layer transition at high free-stream disturbance levels: beyond Klebanoff modes. J. Fluid Mech. 613, 95-124.

GöRTLER, H. 1940 Über eine Dreidimensionale Instabilität Laminarer Grenzschichten an Konkaven Wänden, Nachr. Wiss. Ges. Göttingen Math. Phys. Kl. 2, 1-26.

Gostelow, J. P., Blunden, A. R. \& Walker, G. J. 1994 Effects of free-stream turbulence and adverse pressure gradients on boundary layer transition. J. Turbomach. 116, 392-404.

Herbert, T. 1988 Secondary instability of boundary layers. Annu. Rev. Fluid Mech. 20, 487-526.

Hernon, D., Walsh, E. J. \& McEligot, D. M. 2007 Experimental investigation into the routes to bypass transition and the shear-sheltering phenomenon. J. Fluid Mech. 591, 461-479.

Hilgenfeld, L. \& Pfitzner, M. 2004 Unsteady boundary layer development due to wake-passing effects on a highly loaded linear compressor cascade. J. Turbomach. 126, 493-500.

Hodson, H. P. \& Howell, R. J. 2005 Bladerow interactions, transition, and high-lift aerofoils in low-pressure turbines. Annu. Rev. Fluid Mech. 37, 71-98.

Hughes, J. D. \& WALKer, G. J. 2001 Natural transition phenomena on an axial flow compressor blade. J. Turbomach. 123, 392-401.

JACOBS, R. G. \& Durbin, P. A. 2000 Simulations of bypass transition. J. Fluid Mech. 428, $185-212$.

Jeong, J. \& Hussain, F. 1995 On the identification of a vortex. J. Fluid Mech. 285, 69-94.

Jones, L. E., SANDberg, R. D. \& SANDhaM, N. D. 2008 Direct numerical simulations of forced and unforced separation bubbles on an airfoil at incidence. J. Fluid Mech. 602, 175-207.

Kendall, J. M. 1991 Studies on laminar boundary layer receptivity to free stream turbulence near a leading edge. In FED (ed. Reda et al.), vol. 114, pp. 23-30. ASME.

Klebanoff, P. S., Tidstrom, K. D. \& Sargent, L. M. 1962 The three-dimensional nature of boundary layer instability. J. Fluid Mech. 12, 1-24.

Kleiser, L. \& ZANG, T. A. 1991 Numerical simulation of transition in wall-bounded shear flows. Annu. Rev. Fluid Mech. 23, 495-537.

Leib, S. J., Wundrow, D. W. \& Goldstein, M. E. 1999 Effect of free-stream turbulence and other vortical disturbances on a laminar boundary layer. J. Fluid Mech. 380, 169-203.

LIU, Y., ZAKI, T. A. \& DURBIN, P. A. 2008 a Boundary layer transition by interaction of discrete and continuous modes. J. Fluid Mech. 604, 199-233.

LiU, Y., ZAKI, T. A. \& Durbin, P. A. $2008 b$ Floquet analysis of secondary instability of boundary layers distorted by Klebanoff streaks and Tollmien-Schlichting waves. Phys. Fluids 20, 124102.

LUCHINI, P. 2000 Reynolds-number-independent instability of the boundary layer over a flat surface: optimal perturbations. J. Fluid Mech. 404, 289-309.

Matsubara, M. \& Alfredsson, P. 2001 Disturbance growth in boundary layers subjected to free-stream turbulence. J. Fluid Mech. 430, 149-168.

Morkovin, M. V. 1969 The many faces of transition. In Viscous Drag Reduction (ed. C. S. Wells), pp. 1-31. Plenum Press.

Nagarajan, S., Lele, S. K. \& Ferziger, J. H. 2007 Leading edge effects in bypass transition. J. Fluid Mech. 572, 471-504.

Ovchinnikov, V., Choudhari, M. M. \& Piomelli, U. 2008 Numerical simulations of boundarylayer bypass transition due to high-amplitude free-stream turbulence. J. Fluid Mech. 613, $135-169$.

Phillips, O. M. 1969 Shear-flow turbulence. Annu. Rev. Fluid Mech. 1, 245-264.

Rosenfeld, M., KWAK, D. \& VINOKUR, M. 1991 A fractional step solution method for the unsteady incompressible Navier-Stokes equations in generalized coordinate systems. J. Comput. Phys. 94, $102-137$.

SARIC, W. S. 1994 Görtler vortices. Annu. Rev. Fluid Mech. 26, 379-409.

Saric, W. S., Reed, H. L. \& Kerschen, E. J. 2002 Boundary-layer receptivity to freestream disturbances. Annu. Rev. Fluid Mech. 34 (1), 291-319.

Schreiber, H. A., Steinert, W., Sonoda, T. \& Arima, T. 2004 Advanced high turning compressor airfoils for low Reynolds number condition. Part II: Experimental and numerical analysis. J. Turbomach. 126 (4), 482-492.

Sonoda, T., Yamaguchi, Y., Arima, T., Olhofer, M., Sendhoff, B. \& Schreiber, H.-A. 2004 Advanced high turning compressor airfoils for low Reynolds number condition. Part I. Design and optimization. J. Turbomach. 126 (3), 350-359. 
Spalart, P. R. \& Strelets, M. K. H. 2000 Mechanisms of transition and heat transfer in a separation bubble. J. Fluid Mech. 403, 329-349.

Westin, K. J. A., Boiko, A. V., Klingmann, B. G. B., Kozlov, V. V. \& Alfredsson, P. H. 1994 Experiments in a boundary layer subjected to freestream turbulence. Part 1. Boundary layer structure and receptivity. J. Fluid Mech. 281, 193-218.

Wissink, J. G. 2003 DNS of separating, low Reynolds number flow in a turbine cascade with incoming wakes. Intl J. Heat Fluid Flow 24 (4), 626-635.

Wissink, J. G. \& RoDI, W. 2006 Direct numerical simulation of flow and heat transfer in a turbine cascade with incoming wakes. J. Fluid Mech. 569, 209-347.

Wissink, J. G., Rodi, W. \& Hodson, H. P. 2006 The influence of disturbances carried by periodically incoming wakes on the separating flow around a turbine blade. Intl J. Heat Fluid Flow 27, 721-729.

WU, X. 2001 Receptivity of boundary layers with distributed roughness to vortical and acoustic disturbances: a second-order asymptotic theory and comparison with experiments. J. Fluid Mech. 431, 91-133.

Wu, X. \& Choudhari, M. 2003 Linear and nonlinear instabilities of a Blasius boundary layer perturbed by streamwise vortices. Part 2 . Intermittent instability induced by long-wavelength Klebanoff modes. J. Fluid Mech. 483, 249-286.

Wu, X. \& Durbin, P. A. 2001 Evidence of longitudinal vortices evolved from distorted wakes in a turbine passage. J. Fluid Mech. 446, 199-228.

Wu, X., Jacobs, R. G., Durbin, P. A. \& Hunt, J. 1999 Simulation of boundary layer transition induced by periodically passing wakes. J. Fluid Mech. 398, 109-153.

ZAKI, T. A. \& Durbin, P. A. 2005 Mode interaction and the bypass route to transition. J. Fluid Mech. 531, 85-111.

ZAKI, T. A. \& DURbin, P. A. 2006 Continuous mode transition and the effects of pressure gradient. J. Fluid Mech. 563, 357-388.

ZAKI, T. A. \& SAHA, S. 2009 On shear sheltering and the structure of vortical modes in single- and two-fluid boundary layers. J. Fluid Mech. 626, 111-147.

Zaki, T. A., Wissink, J. G., Durbin, P. A. \& Rodi, W. 2009 Direct computations of boundary layers distorted by migrating wakes in a linear compressor cascade. Flow Turbulence Combust. 83, 307-322. 